\title{
Dynamic Server Allocation Over Time-Varying Channels With Switchover Delay
}

\author{
Güner D. Çelik, Student Member, IEEE, Long B. Le, Member, IEEE, and Eytan Modiano, Fellow, IEEE
}

\begin{abstract}
We consider a dynamic server allocation problem over parallel queues with randomly varying connectivity and server switchover delay between the queues. At each time slot, the server decides either to stay with the current queue or switch to another queue based on the current connectivity and the queue length information. Switchover delay occurs in many telecommunications applications and is a new modeling component of this problem that has not been previously addressed. We show that the simultaneous presence of randomly varying connectivity and switchover delay changes the system stability region and the structure of optimal policies. In the first part of this paper, we consider a system of two parallel queues, and develop a novel approach to explicitly characterize the stability region of the system using state-action frequencies which are stationary solutions to a Markov decision process formulation. We then develop a frame-based dynamic control (FBDC) policy, based on the state-action frequencies, and show that it is throughput optimal asymptotically in the frame length. The FBDC policy is applicable to a broad class of network control systems and provides a new framework for developing throughput-optimal network control policies using state-action frequencies. Furthermore, we develop simple myopic policies that provably achieve more than $90 \%$ of the stability region. In the second part of this paper, we extend our results to systems with an arbitrary finite number of queues. In particular, we show that the stability region characterization in terms of state-action frequencies and the throughput optimality of the FBDC policy follows for the general case. Furthermore, we characterize an outer bound on the stability region and an upper bound on sum throughput and show that a simple myopic policy can achieve this sum-throughput upper bound in the corresponding saturated system. Finally, simulation results show that the myopic policies may achieve the full stability region and are more delay efficient than the FBDC policy in most cases.
\end{abstract}

Index Terms-Downlink, Markov decision process (MDP), queuing, randomly varying connectivity, scheduling, switching delay, switchover delay, uplink, wireless networks.

\section{INTRODUCTION}

$\mathbf{S}$ CHEDULING a dynamic server over time-varying wireless channels is an important and well-studied research problem which provides useful mathematical modeling for many practical applications [7], [17], [24], [28], [31], [32], [37]-[39], [43],

Manuscript received June 08, 2011; revised March 21, 2012; accepted May 22, 2012. Date of publication July 10, 2012; date of current version August 14,2012 . This work was supported in part by the National Science Foundation under Grants CNS-0626781 and CNS-0915988 and in part by the ARO Muri under Grant W911NF-08-1-0238. This paper was presented in part at the 2011 IEEE Conference on Computer Communications, Shanghai, China.

G. D. Çelik and E. Modiano are with the Laboratory for Information and Decision Systems, Massachusetts Institute of Technology, Cambridge, MA 02139 USA (e-mail: gcelik@mit.edu; modiano@mit.edu).

L. B. Le is with the Institut National de la Recherche Scientifique-Énergie, Matériaux et Télécommunications, University of Quebec, Montréal, QC H5A 1K6 Canada (e-mail: long.le@emt.inrs.ca).

Communicated by M. Franceschetti, Associate Editor for Communication Networks.

Color versions of one or more of the figures in this paper are available online at http://ieeexplore.ieee.org.

Digital Object Identifier 10.1109/TIT.2012.2203294

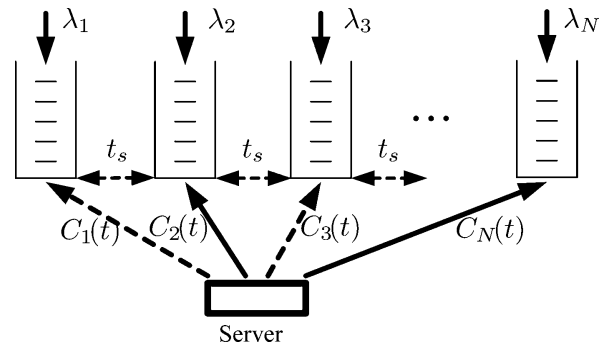

Fig. 1. System model. Parallel queues with randomly varying connectivity processes $C_{1}(t), C_{2}(t), \ldots, C_{N}(t)$ and $t_{s}=1$ slot switchover time.

[44]. However, to the best of our knowledge, the joint effects of randomly varying connectivity and server switchover delay have not been considered previously. In fact, switchover delay is a widespread phenomenon that can be observed in many practical network systems. In satellite systems where a mechanically steered antenna is providing service to ground stations, the time to switch from one station to another can be around $10 \mathrm{~ms}$ [9], [40]. Similarly, the delay for electronic beamforming can be more than $300 \mu \mathrm{s}$ in wireless radio systems [3], [9], [40], and in optical communication systems tuning delay for transceivers can take significant time (microsecond to millisecond) [11], [27].

We consider a dynamic server control problem for parallel queues with time-varying channels and server switchover delay as shown in Fig. 1. We consider a slotted system where the slot length is equal to a single packet transmission time and it takes one slot for the server to switch from one queue to another. ${ }^{1}$ One packet is successfully received from queue $i$ if the server is currently at queue $i$, it decides to stay at queue $i$, and queue $i$ is connected, where $i \in\{1,2, \ldots, N\}$ and $N$ is the total number of queues. The server dynamically decides to stay with the current queue or switch to another queue based on the connectivity and the queue length information. Our goal is to study the impact of the simultaneous presence of switchover delays and randomly varying connectivity on system stability and to develop optimal control algorithms. We show that the stability region changes as a function of the memory in the channel processes, and it is significantly reduced as compared to systems without switchover delay. Furthermore, we show that throughput-optimal policies take a very different structure from the celebrated max-weight (MW) algorithm or its variants.

\section{A. Main Results}

In the first part of this paper, we consider a two-queue system and develop fundamental insights for the problem. We first consider the case of memoryless (i.i.d.) channels where we charac-

\footnotetext{
${ }^{1}$ In a slotted system, even a minimal switchover delay will lead to a loss of a slot due to synchronization issues.
} 
terize the stability region explicitly and show that simple exhaustive-type policies that ignore the current queue size and channel state information are throughput optimal. Next, we consider the Gilbert-Elliot channel model [1], [19] which is a commonly used model to abstract physical channels with memory. We develop a novel methodology to characterize the stability region of the system using state-action frequencies which are steady-state solutions to an Markov decision process (MDP) formulation for the corresponding saturated system, and characterize the stability region explicitly in terms of the connectivity parameters. Using this state-action frequency approach, we develop a novel frame-based dynamic control (FBDC) policy and show that it is throughput optimal asymptotically in the frame length. Our FBDC policy is the only known policy to stabilize systems with randomly varying connectivity and switchover delay and it is novel in that it utilizes the state-action frequencies of the MDP formulation in a dynamic queuing system. Moreover, we develop a simple 1-lookahead myopic (OLM) policy that provably achieves at least $90 \%$ of the stability region, and myopic policies with 2 and 3 lookahead that achieve more than $94 \%$ and $96 \%$ of the stability region, respectively. Finally, we present simulation results suggesting that the myopic policies may be throughput optimal and more delay efficient than the FBDC policy.

In the second part of this paper, we consider the general model with arbitrary finite number of parallel queues. For memoryless (i.i.d.) channel processes, we explicitly characterize the stability region and the throughput-optimal policy. For channels with memory, we show that the stability region characterization in terms of state-action frequencies extends to the general case and establishes a tight outer bound on the stability region and an upper bound on the sum throughput explicitly in terms of the connectivity parameters. We quantify the switching loss in sum throughput as compared to the system with no switchover delays and show that simple myopic policies achieve the sum-throughput upper bound in the corresponding saturated system. We also show that the throughput optimality of the FBDC policy extend to the general case. In fact, the FBDC policy provides a new framework for achieving throughput-optimal network control by applying the state-action frequencies of the corresponding saturated system over frames in the dynamic queuing system. The FBDC policy is applicable to a broad class of systems whose corresponding saturated model is Markovian with a weakly communicating and finite state space, for example, systems with arbitrary switchover delays (i.e., systems that take a fixed, finite number of time slots for switching the server from a given queue to another queue) and general Markov modulated channel processes. Moreover, the framework of the FBDC policy can be utilized to achieve throughput optimality in systems without switchover delay, for instance, in classical network control problems such as those considered in [32], [35], [39], and [44].

\section{B. Related Work}

Optimal control of queuing systems and communication networks has been a very active research topic over the past two decades [17], [24], [28], [31], [32], [37]-[39], [43], [44]. In the seminal paper [38], Tassiulas and Ephremides characterized the stability region of multihop wireless networks and proposed the throughput-optimal MW scheduling algorithm. In [39], the same authors considered a parallel queuing system with randomly varying connectivity where they characterized the stability region of the system explicitly and proved the throughput optimality of the longest connected queue scheduling policy. These results were later extended to joint power allocation and routing in wireless networks in [31] and [32] and optimal scheduling for switches in [35] and [37]. More recently, suboptimal distributed scheduling algorithms with throughput guarantees have been studied in [12], [21], [24] and [43], while the authors in [17] and [28] developed distributed algorithms that achieve throughput optimality (see [18] and[29] for a detailed review). The effect of delayed channel state information was considered in [20], [36] and [44] which showed that the stability region is reduced and that a policy similar to the MW algorithm is throughput optimal.

Perhaps, the closest problem to ours is that of dynamic server allocation over parallel channels with randomly varying connectivity and limited channel sensing that has been investigated in [1], [23] and [45] under the Gilbert-Elliot channel model. The saturated system was considered and the optimality of a myopic policy was established for a single server and two channels in [45], for arbitrary number of channels in [1], and for arbitrary number of channels and servers in [2]. The problem of maximizing throughput in the network while meeting average delay constraints for a small subset of users was considered in [30]. The average delay constraints were turned into penalty functions and the theory of stochastic shortest path problems, which is used for solving dynamic programs with certain special structures, was utilized to minimize the resulting drift+penalty terms. Finally, a partially observable MDP model was used in [16] to analyze dynamic multichannel access in cognitive radio systems. However, none of these existing works consider the server switchover delays.

Switchover delay has been considered in Polling models in queuing theory community (e.g., [6], [22], [25], and [41]); however, randomly varying connectivity was not considered since it may not arise in classical Polling applications. Similarly, scheduling in optical networks under reconfiguration delay was considered in [11] and [15], again in the absence of randomly varying connectivity, where the transmitters and receivers were assumed to be unavailable during reconfiguration. A detailed survey of the work in this field can be found in [41]. To the best of our knowledge, this paper is the first to simultaneously consider random connectivity and server switchover times.

\section{Main Contribution and Organization}

The main contribution of this paper is to solve the scheduling problem in parallel queues with randomly varying connectivity and server switchover delays for the first time. For this, the paper provides a novel framework for solving network control problems via characterizing the stability region in terms of state-action frequencies and achieving throughput optimality by utilizing the state-action frequencies over frames.

This paper is organized as follows. We consider the twoqueue system in Section II where we characterize the stability 


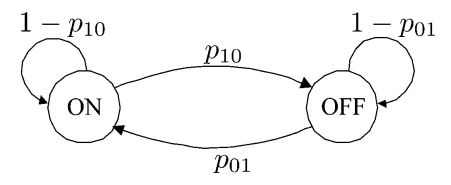

Fig. 2. Markov modulated ON/OFF channel process. We have $p_{10}+p_{01}<$ $1(\epsilon<0.5)$ for positive correlation.

region together with the throughput-optimal policy for memoryless channels. We develop the state-action frequency framework in Section II-C for channels with memory and use it to explicitly characterize the system stability region. We prove the throughput optimality of the FBDC policy in Section II-D and analyze simple myopic policies in Section II-E. We extend our results to the general case in Section III where we also develop outer bounds on the stability region and an upper bound on the sum throughput achieved by a simple myopic policy. We present simulation results in Section IV and conclude this paper in Section V.

\section{Two-Queue System}

\section{A. System Model}

Consider two parallel queues with time-varying channels and one server receiving data packets from the queues. Time is slotted into unit-length time slots equal to one packet transmission time; $t \in\{0,1,2, \ldots\}$. It takes one slot for the server to switch from one queue to the other, and $m(t)$ denotes the queue at which the server is present at slot $t$. Let the i.i.d. stochastic process $A_{i}(t)$ with average arrival rate $\lambda_{i}$ denote the number of packets arriving to queue $i$ at time slot $t$, where $\mathbb{E}\left[A_{i}^{2}(t)\right] \leq A_{\max }^{2}, \quad i \in\{1,2\}$. Let $\mathbf{C}(t)=\left(C_{1}(t), C_{2}(t)\right)$ be the channel (connectivity) process at time slot $t$, where $C_{i}(t)=0$ for the OFF state (disconnected) and $C_{i}(t)=1$ for the ON state (connected). We assume that the processes $A_{1}(t)$, $A_{2}(t), C_{1}(t)$, and $C_{2}(t)$ are independent.

The process $C_{i}(t), \quad i \in\{1,2\}$, is assumed to form the two-state Markov chain with transition probabilities $p_{10}$ and $p_{01}$ as shown in Fig. 2, i.e., the Gilbert-Elliot channel model [1], [19], [23], [45], [46]. The Gilbert-Elliot channel model has been commonly used in modeling and analysis of wireless channels with memory [1], [23], [42], [45], [46]. For ease of exposition, we present the analysis in this section for the symmetric Gilbert-Elliot channel model, i.e., $p_{10}=p_{01}=\epsilon$, and we state the corresponding results for the nonsymmetric case in Appendix B. The steady-state probability of each channel state is equal to 0.5 in the symmetric Gilbert-Elliot channel model. Moreover, for $\epsilon=0.5$, $C_{i}(t)=1$, with probability (w.p.) 0.5 , independently and identically distributed (i.i.d.) at each time slot. We refer to this case as the memoryless channels case.

Let $\mathbf{Q}(t)=\left(Q_{1}(t), Q_{2}(t)\right)$ be the queue lengths at time slot $t$. We assume that $\mathbf{Q}(t)$ and $\mathbf{C}(t)$ are known to the server at the beginning of each time slot. Let $a_{t} \in\{0,1\}$ denote the action taken at the beginning of slot $t$, where $a_{t}=1$ if the server stays with the current queue and $a_{t}=0$ if it switches to the other queue. One packet is successfully received from queue $i$ at time slot $t$, if $m(t)=i, a_{t}=1$, and $C_{i}(t)=1$.
Definition 1 (Stability [29], [31]): The system is stable if

$$
\limsup _{t \rightarrow \infty} \frac{1}{t} \sum_{\tau=0}^{t-1} \sum_{i \in\{1,2\}} \mathbb{E}\left[Q_{i}(\tau)\right]<\infty .
$$

For the case of integer-valued arrival processes, this stability criterion implies the existence of a long run stationary distribution for the queue sizes with bounded first moments [29].

Definition 2 (Stability Region [29], [31]): The stability region $\Lambda$ is the set of all arrival rate vectors $\lambda=\left(\lambda_{1}, \lambda_{2}\right)$ such that there exists a control algorithm that stabilizes the system. The $\delta$-stripped stability region is defined for some $\delta>0$ as $\boldsymbol{\Lambda}^{\delta} \doteq\left\{\left(\lambda_{1}, \lambda_{2}\right) \mid\left(\lambda_{1}+\delta, \lambda_{2}+\delta\right) \in \boldsymbol{\Lambda}\right\}$. A policy is said to achieve $\gamma$-fraction of $\Lambda$, if it stabilizes the system for all input rates inside $\gamma \boldsymbol{\Lambda}$, where $\gamma=1$ for a throughput-optimal policy.

In the following, we start by explicitly characterizing the stability region for both memoryless channels and channels with memory and show that channel memory can be exploited to enlarge the stability region significantly.

\section{B. Motivation: Channels Without Memory}

In this section, we assume that $\epsilon=0.5$ so that the channel processes are i.i.d. over time. The stability region of the corresponding system with no switchover time was established in [39]: $\lambda_{1}, \lambda_{2} \in[0,0.5]$ and $\lambda_{1}+\lambda_{2} \leq 0.75$. Note that when the switchover time is zero, the stability region is the same for both i.i.d. and Markovian channels, which is a special case of the results in [31]. However, when the switchover time is nonzero, the stability region is reduced considerably. Moreover, simple exhaustive-type policies perform well. Specifically, the gated policy under which the server serves all the packets that are present at a queue upon arrival at the queue is throughput optimal.

Theorem 1: The stability region of the system with i.i.d. channels and one-slot switchover delay is given by

$$
\boldsymbol{\Lambda}=\left\{\left(\lambda_{1}, \lambda_{2}\right) \mid \lambda_{1}+\lambda_{2} \leq 0.5, \lambda_{1}, \lambda_{2} \geq 0\right\} .
$$

In addition, the simple exhaustive (Gated) policy is throughput optimal.

The proof is omitted for brevity but it can be found in our technical report [14]. The basic idea behind the proof is that as soon as the server switches to queue $i$ under some policy, the time to the ON state is a geometric random variable with mean 2 slots, independent of the policy. Therefore, a necessary condition for stability is given by the stability condition for a system without switchover times and i.i.d. service times with geometric distribution of mean 2 slots as given by (1). The fact that the simple gated policy is throughput optimal follows from the observation that as the arrival rates are close to the boundary of the stability region, the fraction of time the server spends receiving packets dominates the fraction of time spent on switching [14], [41].

As depicted in Fig. 3, the stability region of the system is considerably reduced for nonzero switchover delay. Note that for systems in which channels are always connected, the stability region is given by $\lambda_{1}+\lambda_{2} \leq 1, \lambda_{1}, \lambda_{2} \geq 0$ and is not affected by the switchover delay [41]. Therefore, it is the combination of 


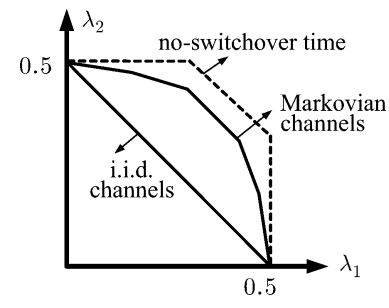

Fig. 3. Stability region under memoryless (i.i.d.) channels and channels with memory (Markovian with $\epsilon<0.5$ ) with and without switchover delay.

switchover delay and random connectivity that result in fundamental changes in system stability.

Remark 1: The results in this section can easily be generalized to the case of nonsymmetric Gilbert-Elliot channels with arbitrary switchover delays. For a system of two queues with arbitrary switchover delays and i.i.d. channels with probabilities $p_{i}, i \in\{1,2\}, \boldsymbol{\Lambda}$ is the set of all $\lambda \geq 0$ such that $\lambda_{1} / p_{1}+$ $\lambda_{2} / p_{2} \leq 1$ [14]. Moreover, simple exhaustive (gated) policy is throughput optimal.

\section{Channels With Memory-Stability Region}

When switchover times are nonzero, the memory in the channel can be exploited to improve the stability region considerably. Moreover, as $\epsilon \rightarrow 0$, the stability region tends to that achieved by the system with no switchover time and for $0<\epsilon<0.5$ it lies between the stability regions corresponding to the two extreme cases $\epsilon=0.5$ and $\epsilon \rightarrow 0$ as shown in Fig. 3 .

We start by analyzing the corresponding system with saturated queues, i.e., both queues are always nonempty. Let $\boldsymbol{\Lambda}_{s}$ denote the set of all time-average expected departure rates that can be obtained from the two queues in the saturated system under all possible policies that are possibly history dependent, randomized, and nonstationary. We will show that $\boldsymbol{\Lambda}=\boldsymbol{\Lambda}_{s}$. We prove the necessary stability conditions in the following Lemma and establish sufficiency in the next section.

Lemma 1: We have that

$$
\boldsymbol{\Lambda} \subseteq \boldsymbol{\Lambda}_{\mathrm{s}}
$$

The proof is omitted here for brevity and it can be found in [14]. An intuitive explanation is as follows. Given a policy $\pi$ for the dynamic queuing system, consider the saturated system with the same sample path of channel realizations and the same set of actions as policy $\pi$. It is clear that the total number of departures from each queue in the saturated system is no less than that in the dynamic queuing system.

Now, we establish the region $\boldsymbol{\Lambda}_{s}$ by formulating the system dynamics as an MDP. Let $\mathbf{s}_{t}=\left(m(t), C_{1}(t), C_{2}(t)\right) \in S$ denote the system state at time $t$ where $\mathcal{S}$ is the set of all states. Also, let $a_{t} \in \mathcal{A}=\{0,1\}$ denote the action taken at time slot $t$ where $\mathcal{A}$ is the set of all actions at each state. Let $\mathbb{H}(t)=\left.\left.\left[\mathbf{s}_{\tau}\right]\right|_{\tau=0} ^{t} \cup\left[a_{\tau}\right]\right|_{\tau=0} ^{t-1}$ denote the full history of the system state until time $t$ and let $\Upsilon(\mathcal{A})$ denote the set of all probability distributions on $\mathcal{A}$. For the saturated system, a policy is a mapping from the set of all possible past histories to $\Upsilon(\mathcal{A})$ [5], [26]. A policy is said to be stationary if, given a particular state, it applies the same decision rule in all stages and under a stationary policy, the process $\left\{\mathbf{s}_{t} ; t \in \mathbb{N} \cup\{0\}\right\}$ forms a Markov chain. In each time slot $t$, the server observes the current state $\mathbf{s}_{t}$ and chooses an action $a_{t}$. Then, the next state $j$ is realized according to the transition probabilities $\mathbf{P}(j \mid \mathbf{s}, a)$, which depend on the random channel processes. Now, we define the reward functions as follows:

$$
\begin{aligned}
& \bar{r}_{1}(\mathbf{s}, a) \doteq 1 \text { if } \mathbf{s}=(1,1,1) \text { or } \mathbf{s}=(1,1,0), \text { and } a=1 \\
& \bar{r}_{2}(\mathbf{s}, a) \doteq 1 \text { if } \mathbf{s}=(2,1,1) \text { or } \mathbf{s}=(2,0,1), \text { and } a=1
\end{aligned}
$$

and $\bar{r}_{1}(\mathbf{s}, a)=\bar{r}_{2}(\mathbf{s}, a) \doteq 0$ otherwise. That is, a reward is obtained when the server stays at an ON channel. We are interested in the set of all possible time-average expected departure rates; therefore, given some $\alpha_{1}, \alpha_{2} \geq 0, \alpha_{1}+\alpha_{2}=1$, we define the system reward at time $t$ by $\bar{r}(\mathbf{s}, a) \doteq \alpha_{1} \bar{r}_{1}(\mathbf{s}, a)+\alpha_{2} \bar{r}_{2}(\mathbf{s}, a)$. The average reward of policy $\pi$ is defined as follows:

$$
r^{\pi} \doteq \limsup _{K \rightarrow \infty} \frac{1}{K} E\left\{\sum_{t=1}^{K} \bar{r}\left(\mathbf{s}_{t}, a_{t}^{\pi}\right)\right\} .
$$

Given some $\alpha_{1}, \alpha_{2} \geq 0$, we are interested in the policy that achieves the maximum time-average expected reward $r^{*} \doteq \max _{\pi} r^{\pi}$. This optimization problem is a discrete time MDP characterized by the state transition probabilities $\mathbf{P}(j \mid \mathbf{s}, a)$ with eight states and two actions per state. Furthermore, any given pair of states are accessible from each other (i.e., there exists a positive probability path between the states) under some stationary-deterministic policy. Therefore, this MDP belongs to the class of weakly communicating $\operatorname{MDPs}^{2}[34]$.

State-Action Frequency Approach: For weakly communicating MDPs with finite state and action spaces and bounded rewards, there exists an optimal stationary-deterministic policy, given as a solution to standard Bellman's equation, with optimal average reward independent of the initial state [34, Th. 8.4.5]. This is because if a stationary policy has a nonconstant gain over initial states, one can construct another stationary policy with constant gain which dominates the former policy, which is possible since there exists a positive probability path between any two recurrent states under some stationary policy [26]. The state-action frequency approach, or the dual linear program $(L P)$ approach, given in the following provides a systematic and intuitive framework to solve such average cost MDPs, and it can be derived using Bellman's equation and the monotonicity property of dynamic programs [34, Sec 8.8]

$$
\text { Maximize } \sum_{\mathbf{s} \in \mathcal{S}} \sum_{a \in \mathcal{A}} \bar{r}(\mathbf{s}, a) \mathbf{x}(\mathbf{s}, a)
$$

subject to the balance equations

$$
\mathbf{x}(\mathbf{s} ; 1)+\mathbf{x}(\mathbf{s} ; 0)=\sum_{\mathbf{s}^{\prime} \in \mathcal{S}} \sum_{a \in \mathcal{A}} \mathbf{P}\left(\mathbf{s} \mid \mathbf{s}^{\prime}, a\right) \mathbf{x}\left(\mathbf{s}^{\prime}, a\right), \forall \mathbf{s} \in S
$$

the normalization condition

$$
\sum_{\mathbf{s} \in \mathcal{S}} \mathbf{x}(\mathbf{s} ; 1)+\mathbf{x}(\mathbf{s} ; 0)=1
$$

${ }^{2}$ In fact, other than the trivial suboptimal policy $\pi_{s}$ that decides to stay with the current queue in all states, all stationary-deterministic policies are unichain, namely, they have a single recurrent class regardless of the initial state. Hence, when $\pi_{s}$ is excluded, we have a unichain MDP. 
and the nonnegativity constraints

$$
\mathbf{x}(\mathbf{s}, a) \geq 0, \mathbf{s} \in \mathcal{S}, \in \mathcal{A} .
$$

The feasible region of this LP constitutes a polytope called the state-action polytope $\mathbf{X}$ and the elements of this polytope $\mathbf{X} \in \mathbf{X}$ are called state-action frequency vectors. Clearly, $\mathbf{X}$ is a convex, bounded, and closed set. Note that $\mathbf{x}(\mathbf{s} ; 1)$ can be interpreted as the stationary probability that action stay is taken at state $\mathbf{s}$. More precisely, a point $\mathbf{x} \in \mathbf{X}$ corresponds to a stationary-randomized policy that takes action $a \in\{0,1\}$ at state $\mathbf{s}$ w.p.

$$
\mathbf{P}(\text { action } a \text { at state } \mathbf{s})=\frac{\mathbf{x}(\mathbf{s}, a)}{\mathbf{x}(\mathbf{s} ; 1)+\mathbf{x}(\mathbf{s} ; 0)}, a \in A, \mathbf{s} \in S_{x}
$$

where $S_{x}$ is the set of recurrent states given by $S_{x} \equiv\{\mathbf{s} \in S$ : $\mathbf{x}(\mathbf{s} ; 1)+\mathbf{x}(\mathbf{s} ; 0)>0\}$, and actions are arbitrary for transient states $\mathbf{s} \in S / S_{x}$ [26], [34].

Next, we argue that the empirical state-action frequencies corresponding to any given policy (possibly randomized, nonstationary, or non-Markovian) lie in the state-action polytope $\mathbf{X}$. This ensures us that the optimal solution to the dual LP in (4) is over possibly nonstationary and history-dependent policies. In the following, we give the precise definition and the properties of the set of empirical state-action frequencies. We define the empirical state-action frequencies $\hat{x}^{T}(\mathbf{s}, a)$ as

$$
\hat{x}^{T}(\mathbf{s}, a) \doteq \frac{1}{T} \sum_{t=1}^{T} I_{\left\{\mathbf{s}_{t}=\mathbf{s}, a_{t}=a\right\}}
$$

where $I_{E}$ is the indicator function of an event $E$, i.e., $I_{E}=1$ if $E$ occurs and $I_{E}=0$ otherwise. Given a policy $\pi$, let $P^{\pi}$ be the state transition probabilities under the policy $\pi$ and $\phi=\left(\phi_{s}\right)$ an initial state distribution with $\sum_{\mathbf{s} \in \mathcal{S}} \phi_{\mathbf{s}}=1$. We let $x_{\pi, \phi}^{T}(\mathbf{s}, a)$ be the expected empirical state-action frequencies under policy $\pi$ and initial state distribution $\phi$

$$
\begin{aligned}
x_{\pi, \phi}^{T}(\mathbf{s}, a) & \doteq \mathbb{E}^{\pi, \phi}\left[\hat{x}^{T}(\mathbf{s}, a)\right] \\
& =\frac{1}{T} \sum_{t=1}^{T} \sum_{\mathbf{s}^{\prime} \in \mathcal{S}} \phi_{\mathbf{s}^{\prime}} P^{\pi}\left(\mathbf{s}_{t}=\mathbf{s}, a_{t}=a \mid \mathbf{s}_{0}=\mathbf{s}^{\prime}\right) .
\end{aligned}
$$

We let $\mathbf{x}_{\pi, \phi} \in \Upsilon(\mathcal{S} \times \mathcal{A})$ (as in [26] and [34]) be the limiting expected state-action frequency vector, if it exists, starting from an initial state distribution $\phi$, under a general policy $\pi$ (possibly randomized, nonstationary, or non-Markovian):

$$
x_{\pi, \phi}(\mathbf{s}, a)=\lim _{T \rightarrow \infty} x_{\pi, \phi}^{T}(\mathbf{s}, a) .
$$

Let the set of all limit points be defined by

$$
\begin{aligned}
\mathbf{X}_{\Pi}^{\phi} \doteq & \{x \in \Upsilon(\mathcal{S} \times \mathcal{A}): \text { there exists a policy } \pi \text { s.t. } \\
& \text { the limit in } \left.(10) \text { exists and } \mathbf{x}=\mathbf{x}_{\pi, \phi}\right\} .
\end{aligned}
$$

Similarly, let $X_{\Pi^{\prime}}^{\phi}$ denote the set of all limit points of a particular class of policies $\Pi^{\prime}$, starting from an initial state distribution $\phi$. We let $\Pi_{S D}$ denote the set of all stationary-deterministic policies and we let $\operatorname{co}(\Xi)$ denote the closed convex hull of set $\Xi$. The following theorem establishes the equivalency between the set of all achievable limiting state-action frequencies and the state-action polytope.

Theorem 2: [26, Th. 3.1], [34, Th. 8.9.3]: For any initial state distribution $\phi$

$$
\operatorname{co}\left(\mathbf{X}_{\Pi_{S D}}^{\phi}\right)=\mathbf{X}_{\Pi}^{\phi}=\mathbf{X} .
$$

We have $c o\left(\mathbf{X}_{\Pi_{S D}}^{\phi}\right) \subseteq \mathbf{X}_{\Pi}^{\phi}$ since convex combinations of vectors in $\mathbf{X}_{\Pi_{S D}}^{\phi}$ correspond to limiting expected state-action frequencies for stationary-randomized policies, which can also be obtained by time sharing between stationary-deterministic policies. The inverse relation $c o\left(\mathbf{X}_{\Pi_{S D}}^{\phi}\right) \supseteq \mathbf{X}_{\Pi}^{\phi}$ holds since for weakly communicating MDPs, there exists a stationary-deterministic optimal policy independent of the initial state distribution. Next, for any stationary-deterministic policy, the underlying Markov chain is stationary, and therefore, the limits $\mathbf{x}_{\pi, \phi}$ exists and satisfies the constraints (5)-(7) of the polytope $\mathbf{X}$. Using $c o\left(\mathbf{X}_{\Pi_{S D}}^{\phi}\right)=\mathbf{X}_{\Pi}^{\phi}$ and the convexity of $\mathbf{X}$ establishes $\mathbf{X}_{\Pi}^{\phi} \subseteq \mathbf{X}$. Furthermore, via (8), every $\mathbf{x} \in \mathbf{X}$ corresponds to a stationary-randomized policy for which the limits $\mathbf{x}_{\pi, \phi}$ exists, establishing $\mathbf{X}_{\Pi}^{\phi} \supseteq \mathbf{X}$.

Letting $\operatorname{ext}(\mathbf{X})$ denote the set of extreme (corner) points of $\mathbf{X}$, an immediate corollary to Theorem 2 is as follows.

Corollary 1: [26], [34]: For any initial state distribution $\phi$

$$
\operatorname{ext}(\mathbf{X})=\mathbf{X}_{\Pi_{S D}}^{\phi} .
$$

The intuition behind this corollary is that if $\mathbf{x}$ is a corner point of $\mathbf{X}$, it cannot be expressed as a convex combination of any two other elements in $\mathbf{X}$; therefore, for each state $\mathbf{~ s , ~ o n l y ~ o n e ~ a c t i o n ~}$ has a nonzero probability.

Finally, we have that under any policy, the probability of a large distance between the empirical expected state-action frequency vectors and the state-action polytope $\mathbf{X}$ decays exponentially fast in time. This result is similar to the mixing time of an underlying Markov chain to its steady state and we utilize such convergence results within the Lyapunov drift analysis for the dynamic queuing system in Section II-D.

Rate Polytope $\boldsymbol{\Lambda}_{s}$ : Using the theory on state-action polytopes in the previous section, we characterize the set of all achievable time-average expected rates in the saturated system, $\boldsymbol{\Lambda}_{s}$. The following linear transformation of the state-action polytope $\mathbf{X}$ defines the 2-D rate polytope [26]

$$
\begin{aligned}
\boldsymbol{\Lambda}_{s}=\left\{\left(r_{1}, r_{2}\right) \mid r_{1}\right. & =\sum_{\mathbf{s} \in \mathcal{S}} \sum_{a \in \mathcal{A}} \mathbf{x}(\mathbf{s}, a) \bar{r}_{1}(\mathbf{s}, a) \\
r_{2} & \left.=\sum_{\mathbf{s} \in \mathcal{S}} \sum_{a \in \mathcal{A}} \mathbf{x}(\mathbf{s}, a) \bar{r}_{2}(\mathbf{s}, a), \mathbf{x} \in \mathbf{X}\right\}
\end{aligned}
$$

where $\bar{r}_{1}(\mathbf{s}, a)$ and $\bar{r}_{2}(\mathbf{s}, a)$ are the reward functions defined in (2) and (3). This polytope is the set of all time-average expected departure rate pairs that can be obtained in the saturated system, i.e., it is the rate region $\boldsymbol{\Lambda}_{s}$. An explicit way of characterizing $\boldsymbol{\Lambda}_{s}$ is given in Algorithm 1. 
Algorithm 1 Stability Region Characterization

1:Given $\alpha_{1}, \alpha_{2} \geq 0, \alpha_{1}+\alpha_{2}=1$, solve the following Linear Program (LP)

$$
\begin{array}{ll}
\max . & \alpha_{1} r_{1}(\mathbf{x})+\alpha_{2} r_{2}(\mathbf{x}) \\
& \text { subject to } \quad \mathbf{x} \in \mathbf{X} .
\end{array}
$$

2:For a given $\alpha_{2} / \alpha_{1}$ ratio, there exists an optimal solution $\left(r_{1}^{*}, r_{2}^{*}\right)$ of the LP in (11) at a corner point of $\boldsymbol{\Lambda}_{\mathbf{s}}$. Find all possible corner points and take their convex combination.

The fundamental theorem of linear programming guarantees that an optimal solution of the LP in (11) lies at a corner (extreme) point of the polytope $\mathbf{X}$ [8]. Furthermore, the one-to-one correspondence between the extreme points of the polytope $\mathbf{X}$ and stationary-deterministic polices stated in Corollary 1 is useful for finding the solutions of the earlier LP for all possible $\alpha_{2} / \alpha_{1}$ ratios. Namely, there are a total of $2^{8}$ stationary-deterministic policies since we have eight states and two actions per state and finding the rate pairs corresponding to these 256 stationary-deterministic policies and taking their convex combination gives $\boldsymbol{\Lambda}_{s}$. Fortunately, we do not have to go through this tedious procedure. The fact that at a vertex of (11) either $\mathbf{x}(\mathbf{s} ; 1)$ or $\mathbf{x}(\mathbf{s} ; 0)$ has to be zero for each $\mathbf{s} \in \mathbf{S}$ provides a useful guideline for analytically solving this LP. The following theorem characterizes the stability region explicitly. It shows that the stability region enlarges as the channel has more memory and that there is a critical value of the channel correlation parameter given by $\epsilon_{c} \doteq 1-\sqrt{2} / 2$ at which the structure of the stability region changes.

Theorem 3: The rate region $\Lambda_{s}$ is the set of all rates $r_{1} \geq 0$, $r_{2} \geq 0$ that for $\epsilon<\epsilon_{c}$ satisfy

$$
\begin{aligned}
\epsilon r_{1}+(1-\epsilon)^{2} r_{2} & \leq \frac{(1-\epsilon)^{2}}{2} \\
(1-\epsilon) r_{1}+\left(1+\epsilon-\epsilon^{2}\right) r_{2} & \leq \frac{3}{4}-\frac{\epsilon}{2} \\
r_{1}+r_{2} & \leq \frac{3}{4}-\frac{\epsilon}{2} \\
\left(1+\epsilon-\epsilon^{2}\right) r_{1}+(1-\epsilon) r_{2} & \leq \frac{3}{4}-\frac{\epsilon}{2} \\
(1-\epsilon)^{2} r_{1}+\epsilon r_{2} & \leq \frac{(1-\epsilon)^{2}}{2}
\end{aligned}
$$

and for $\epsilon \geq \epsilon_{c}$ satisfy

$$
\begin{aligned}
r_{1}+(1-\epsilon)(3-2 \epsilon) r_{2} & \leq \frac{(1-\epsilon)(3-2 \epsilon)}{2} \\
r_{1}+r_{2} & \leq \frac{3}{4}-\frac{\epsilon}{2} \\
(1-\epsilon)(3-2 \epsilon) r_{1}+r_{2} & \leq \frac{(1-\epsilon)(3-2 \epsilon)}{2} .
\end{aligned}
$$

Observation 1: The maximum achievable sum rate in the saturated system is given by

$$
r_{1}+r_{2}=\frac{3}{4}-\frac{\epsilon}{2}
$$

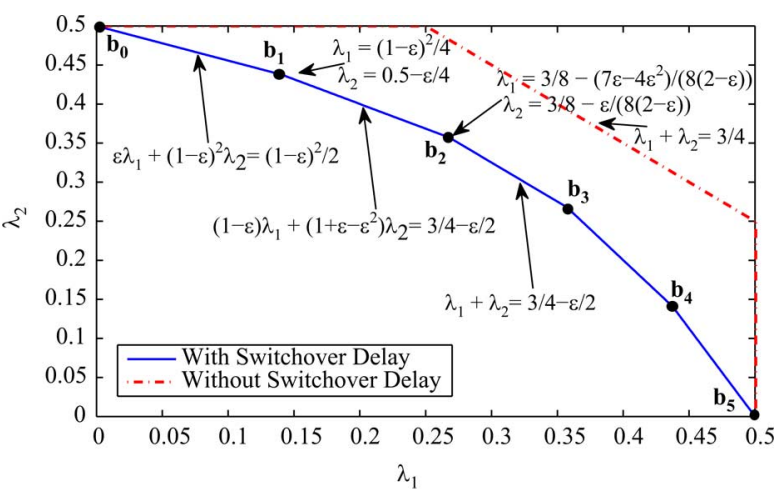

(a)

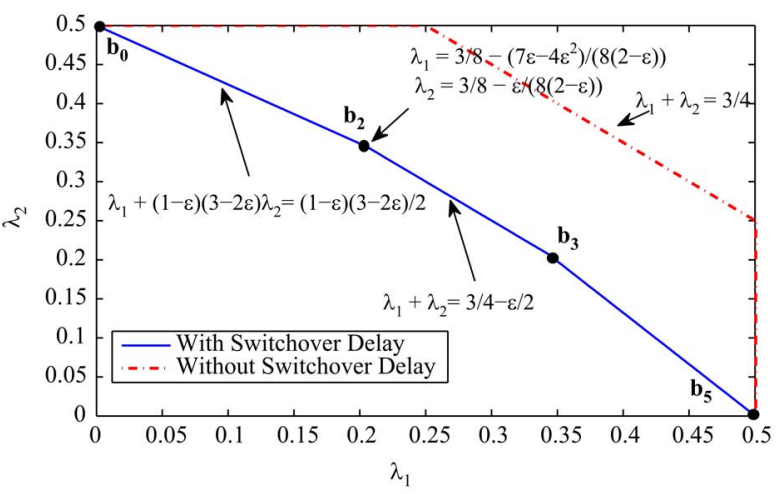

(b)

Fig. 4. Stability region under channels with memory, with and without switchover delay for (a) $\epsilon=0.25<\epsilon_{c}$ and (b) $\epsilon=0.40 \geq \epsilon_{c}$.

The proof of the theorem is lengthy and it is omitted here for brevity; however, it can be found in [14]. Observation 1 is proved in Appendix A and it constitutes an example of how to solve the LP in (11) for the case of, $\alpha_{1}=\alpha_{2}$, i.e., the sum-rate maximization. The proof of Theorem 3 is based on solving the LP in (11) for all weights $\alpha_{1}$ and $\alpha_{2}$ to find the corner points of $\boldsymbol{\Lambda}_{\mathrm{s}}$, and then applying Algorithm 1. This derivation can be found in [14].

Note that $r_{1}+r_{2} \leq \frac{3}{4}$ is the boundary of the stability region for the system without switchover delay analyzed in [39], where the probability that at least one channel is in ON state is $3 / 4$. Therefore, $\epsilon / 2$ is the throughput loss due to the one slot switchover delay. This throughput loss corresponds to the probability that the server is at a queue with an OFF state when the other queue is in an ON state.

The stability regions for the two ranges of $\epsilon$ are displayed in Fig. 4(a) and 4(b). As $\epsilon \rightarrow 0.5$, the stability region converges to that of the i.i.d. channels with $\mathrm{ON}$ probability equal to 0.5 . In this regime, knowledge of the current channel state is of no value. As $\epsilon \rightarrow 0$, the stability region converges to that for the system with no switchover time in [39]. In this regime, the channels are likely to stay the same for many consecutive time slots; therefore, the effect of switching delay is negligible.

The rate region $\boldsymbol{\Lambda}_{s}$ for the case of nonsymmetric Gilbert-Elliot channels is given in Appendix B.

Remark 2: The stability region characterization in terms of state-action frequencies is general. For instance, this technique can be used to establish the stability regions of systems with 
more than two queues, arbitrary switchover times, and more complicated Markovian channel processes. Of course, explicit characterization as in Theorem 3 may not always be possible.

\section{FBDC Policy}

We propose an FBDC policy inspired by the characterization of the stability region in terms of state-action frequencies and prove that it is throughput optimal asymptotically in the frame length. The motivation behind the FBDC policy is that a policy $\pi^{*}$ that achieves the optimization in (11) for given weights $\alpha_{1}$ and $\alpha_{2}$ for the saturated system should achieve a good performance in the original system when the queue sizes $Q_{1}$ and $Q_{2}$ are used as weights. This is because first, the policy $\pi^{*}$ will lead to similar average departure rates in both systems for sufficiently high queue sizes, and second, the usage of queue sizes as weights creates self-adjusting policies that capture the dynamic changes due to stochastic arrivals similar to MW scheduling in [38]. Specifically, we divide the time into equal-size intervals of $T$ slots and let $Q_{1}(j T)$ and $Q_{2}(j T)$ be the queue lengths at the beginning of the $j$ th interval. We find the deterministic policy that optimally solves (11) when $Q_{1}(j T)$ and $Q_{2}(j T)$ are used as weights and then apply this policy in each time slot of the frame. The FBDC policy is described in Algorithm 2 in details.

Algorithm 2 FBDC Policy

1:Find the policy $\pi^{*}$ that optimally solves the following LP:

$$
\begin{array}{cc}
\max \cdot\left\{r_{1}, r_{2}\right\} & Q_{1}(j T) r_{1}+Q_{2}(j T) r_{2} \\
\text { subject to } & \left(r_{1}, r_{2}\right) \in \boldsymbol{\Lambda}_{s}
\end{array}
$$

where $\boldsymbol{\Lambda}_{s}$ is the rate polytope derived in Section II-C.

2:Apply $\pi^{*}$ in each time slot of the frame.

There exists an optimal solution $\left(r_{1}^{*}, r_{2}^{*}\right)$ of the LP in (12) that is a corner point of $\boldsymbol{\Lambda}_{s}[8]$ and the policy $\pi^{*}$ that corresponds to this point is a stationary-deterministic policy by Corollary 1 .

Theorem 4: For any $\delta>0$, there exists a large enough frame length $T$ such that the FBDC policy stabilizes the system for all arrival rates within the $\delta$-stripped stability region $\boldsymbol{\Lambda}_{s}^{\delta}=\boldsymbol{\Lambda}_{s}-\delta \mathbf{1}$.

An immediate corollary to this theorem is as follows.

Corollary 2: The FBDC policy is throughput optimal asymptotically in the frame length.

The proof of Theorem 4 is given in Appendix C. It performs a drift analysis using the standard quadratic Lyapunov function. However, it is novel in utilizing the state-action frequency framework of MDP theory within the Lyapunov drift arguments. The basic idea is that, for sufficiently large queue lengths, when the optimal policy solving (12), $\pi^{*}$, is applied over a sufficiently long frame of $T$ slots, the average output rates of both the actual system and the corresponding saturated system converge to $\mathbf{r}^{*}$. For the saturated system, the probability of a large difference between empirical and steady-state rates decreases exponentially fast in $T$ [26], similar to the convergence of a positive recurrent Markov chain to its steady state. Therefore, for sufficiently large queue lengths, the difference between the empirical rates in the actual system and $r^{*}$ also decreases with $T$.
TABLE I

FBDC POLICY MAPPING From THE QUeUe Sizes TO THE CORNERS OF $\boldsymbol{\Lambda}_{\mathbf{s}}, b_{0}, b_{1}, b_{2}, b_{3}, b_{4}, b_{5}$ SHOWN IN FIG. 4(A), FOR $\epsilon<\epsilon_{c}$. FOR EACH STATE $\mathbf{s}=\left(m(t), C_{1}(t), C_{2}(t)\right)$, THE OPTIMAL ACTION IS SPECIFIED. THE THRESHOLDS ON $Q_{2} / Q_{1}$ ARE $0, T_{1}^{*}=\epsilon /(1-\epsilon)^{2}, T_{2}^{*}=(1-\epsilon) /\left(1+\epsilon-\epsilon^{2}\right)$,

\begin{tabular}{|c|c|c|c|c|c|}
\hline & $T_{1}^{*}(\epsilon)$ & ${ }_{2}^{*}(\epsilon)$ & 1 & $(\epsilon)$ & $4(\epsilon)$ \\
\hline corne & corner $b_{4}$ & corner $b_{3}$ & orner $b_{2}$ & $b_{1}$ & corner $b_{0}$ \\
\hline $\begin{array}{l}(1,1,1): \text { stay } \\
(1,1,0): \text { stay } \\
(1,0,1): \text { stay } \\
(1,0,0): \text { stay } \\
(2,1,1): \text { switch } \\
(2,1,0): \text { switch } \\
(2,0,1): \text { switch } \\
(2,0,0): \text { switch }\end{array}$ & $\begin{array}{l}(1,1,1): \text { stay } \\
(1,1,0): \text { stay } \\
(1,0,1): \text { switch } \\
(1,0,0): \text { stay } \\
(2,1,1): \text { switch } \\
(2,1,0): \text { switch } \\
(2,0,1): \text { stay } \\
(2,0,0): \text { switch }\end{array}$ & $\begin{array}{l}(1,1,1): \text { stay } \\
(1,1,0): \text { stay } \\
(1,0,1): \text { switch } \\
(1,0,0): \text { stay } \\
(2,1,1): \text { stay } \\
(2,1,0): \text { switch } \\
(2,0,1): \text { stay } \\
(2,0,0): \text { switch }\end{array}$ & $\begin{array}{l}(1,1,1): \text { stay } \\
(1,1,0): \text { stay } \\
(1,0,1): \text { switch } \\
(1,0,0): \text { switch } \\
(2,1,1): \text { stay } \\
(2,1,0): \text { switch } \\
(2,0,1): \text { stay } \\
(2,0,0): \text { stay }\end{array}$ & $\begin{array}{l}(1,1,1): \text { switch } \\
(1,1,0): \text { stay } \\
(1,0,1): \text { switch } \\
(1,0,0): \text { switch } \\
(2,1,1): \text { stay } \\
(2,1,0): \text { switch } \\
(2,0,1): \text { stay } \\
(2,0,0): \text { stay }\end{array}$ & $\begin{array}{l}(1,1,1): \text { switch } \\
(1,1,0): \text { switch } \\
(1,0,1): \text { switch } \\
(1,0,0): \text { switch } \\
(2,1,1): \text { stay } \\
(2,1,0): \text { stay } \\
(2,0,1): \text { stay } \\
(2,0,0): \text { stay }\end{array}$ \\
\hline
\end{tabular}
$1, T_{3}^{*}=\left(1+\epsilon-\epsilon^{2}\right) /(1-\epsilon), T_{4}^{*}=(1-\epsilon)^{2} / \epsilon$

TABLE II

FBDC POLICY MAPPING FROM THE QUEUE SIZES TO THE CORNERS OF $\boldsymbol{\Lambda}_{\mathbf{s}}, b_{0}, b_{2}, b_{3}, b_{5}$ SHOWN IN Fig. 4(B), FOR $\epsilon \geq \epsilon_{c}$. FOR EACH STATE $\mathbf{s}=\left(m(t), C_{1}(t), C_{2}(t)\right)$, THE OPTIMAL ACTION IS SPECIFIED. THE THRESHOLDS ON $Q_{2} / Q_{1}$ ARE $0, T_{1}^{*}=1 /((1-\epsilon)(3-2 \epsilon))$,

\begin{tabular}{|c|c|c|c|}
\hline & & & \\
\hline corner $b_{5}$ & corner $b_{3}$ & corner $b_{2}$ & corner $b_{0}$ \\
\hline $\begin{array}{l}(1,1,1): \text { stay } \\
(1,1,0): \text { stay } \\
(1,0,1): \text { stay } \\
(1,0,0): \text { stay } \\
(2,1,1): \text { switch } \\
(2,1,0): \text { switch } \\
(2,0,1): \text { switch } \\
(2,0,0): \text { switch }\end{array}$ & $\begin{array}{l}(1,1,1): \text { stay } \\
(1,1,0): \text { stay } \\
(1,0,1): \text { switch } \\
(1,0,0): \text { stay } \\
(2,1,1): \text { stay } \\
(2,1,0): \text { switch } \\
(2,0,1): \text { stay } \\
(2,0,0): \text { switch }\end{array}$ & $\begin{array}{l}(1,1,1): \text { stay } \\
(1,1,0): \text { stay } \\
(1,0,1): \text { switch } \\
(1,0,0): \text { switch } \\
(2,1,1): \text { stay } \\
(2,1,0) \text { : switch } \\
(2,0,1): \text { stay } \\
(2,0,0): \text { stay }\end{array}$ & $\begin{array}{l}(1,1,1) \text { : switch } \\
(1,1,0): \text { switch } \\
(1,0,1): \text { switch } \\
(1,0,0) \text { : switch } \\
(2,1,1) \text { : stay } \\
(2,1,0) \text { : stay } \\
(2,0,1): \text { stay } \\
(2,0,0) \text { : stay }\end{array}$ \\
\hline
\end{tabular}
$1, T_{2}^{*}=(1-\epsilon)(3-2 \epsilon)$

This ultimately results in a negative Lyapunov drift when $\lambda$ is inside the $\delta(T)$-stripped stability region since from (12), we have $Q_{1}(j T) r_{1}^{*}+Q_{2}(j T) r_{2}^{*}>Q_{1}(j T) \lambda_{1}+Q_{2}(j T) \lambda_{2}$.

The FBDC policy is easy to implement since it does not require the arrival rate information for stabilizing the system for arrival rates in $\boldsymbol{\Lambda}-\delta(T) \mathbf{1}$, and it does not require the solution of the LP (12) for each frame. Instead, one can solve the LP (12) for all possible $\left(Q_{1}, Q_{2}\right)$ pairs only once in advance and create a mapping from $\left(Q_{1}, Q_{2}\right)$ pairs to the corners of the stability region. Then, this mapping can be used to find the corresponding optimal saturated-system policy to be applied during each frame. Solving the LP in (12) for all possible $\left(Q_{1}, Q_{2}\right)$ pairs is possible because first, the solution of the LP will be one of the corner points of the stability region in Fig. 4, and second, the weights $\left(Q_{1}, Q_{2}\right)$, which are the inputs to the LP, determine which corner point is optimal. The theory of linear programming suggests that the solution to the LP in (12) depends only on the relative value of the weights $\left(Q_{1}, Q_{2}\right)$ with respect to each other. Namely, changing the queue size ratio $Q_{2} / Q_{1}$ varies the slope of the objective function of the LP in (12), and the value of this slope $Q_{2} / Q_{1}$ with respect to the slopes of the lines in the stability region in Fig. 4 determines which corner point the FBDC policy operates on. These mappings from the queue size ratios to the corners of the stability region are shown in Table I for the case of $\epsilon<\epsilon_{c}$ and in Table II for the case of $\epsilon \geq \epsilon_{c}$. The corresponding mappings for the FBDC policy for the case of nonsymmetric Gilbert-Elliot channels are shown in Appendix B. 
Given these tables, one no longer needs to solve the LP (12) for each frame, but just has to perform a simple table lookup to determine the optimal policy to use in each frame.

In the next section, we provide an upper bound to the average packet delay under the FBDC policy, which is linear in $T$. This suggest that the packet delay increases with increasing frame lengths as expected. However, such increases are at most linear in $T$. Note that the FBDC policy can also be implemented without any frames by setting $T=1$, i.e., by solving the LP in Algorithm 2 in each time slot. The simulation results in Section IV suggest that the FBDC policy implemented without frames has a similar throughput performance to the original FBDC policy. This is because for large queue lengths, the optimal solution of the LP in (12) depends on the queue length ratios, and hence, the policy $\pi^{*}$ that solves the LP optimally does not change fast when the queue lengths get large. When the policy is implemented without the use of frames, it becomes more adaptive to dynamic changes in the queue lengths, which results in better delay performance than the frame-based implementations. We discuss more properties of the FBDC policy, such as its complexity and implementation tradeoffs in Section III-D1.

Delay Upper Bound: The delay upper bound in this section is easily derived once the stability of the FBDC algorithm is established. The stability proof utilizes the following quadratic Lyapunov function:

$$
L(\mathbf{Q}(t))=\sum_{i=1}^{2} Q_{i}^{2}(t)
$$

which represents a quadratic measure of the total load in the system at time slot $t$. Let $t_{k}$ denote the time slots at the frame boundaries, $k=0,1, \ldots$, and define the $T$-step conditional drift

$$
\Delta_{T}\left(t_{k}\right) \triangleq \mathbb{E}\left[L\left(\mathbf{Q}\left(t_{k}+T\right)\right)-L\left(\mathbf{Q}\left(t_{k}\right)\right) \mid \mathbf{Q}\left(t_{k}\right)\right] .
$$

The following drift expression follows from the stability analysis in Appendix C:

$$
\frac{\Delta_{T}\left(t_{k}\right)}{2 T} \leq B T-\left(\sum_{i} Q_{i}\left(t_{k}\right)\right) \xi
$$

where $B=1+A_{\max }^{2}, \lambda$ is strictly inside the $\delta$-stripped stability region $\Lambda-\delta 1$, and $\xi>0$ represents a measure of the distance of $\lambda$ to the boundary of $\Lambda-\delta 1$. Taking expectations with respect to $\mathbf{Q}\left(t_{k}\right)$, writing a similar expression over the frame boundaries $t_{k}, k \in\{0,1,2, \ldots, K\}$, summing them, and telescoping these expressions lead to

$$
L\left(\mathbf{Q}\left(t_{K}\right)\right)-L(\mathbf{Q}(0)) \leq 2 K B T^{2}-2 \xi T \sum_{k=0}^{K-1} \mathbb{E}\left[\sum_{i} Q_{i}\left(t_{k}\right)\right] .
$$

Using $L\left(\mathbf{Q}\left(t_{K}\right)\right) \geq 0$ and $L(\mathbf{Q}(0))=0$, we have

$$
\limsup _{K \rightarrow \infty} \frac{1}{K} \sum_{k=0}^{K-1} \sum_{i} \mathbb{E}\left[Q_{i}\left(t_{k}\right)\right] \leq \frac{B T}{\xi} .
$$

For $t \in\left(t_{k}, t_{k+1}\right)$, we have $Q_{i}(t) \leq Q_{i}\left(t_{k}\right)+\sum_{\tau=0}^{T-1} A_{i}\left(t_{k}+\tau\right)$. Upon taking expectations, we have $\mathbb{E}\left[Q_{i}(t)\right] \leq \mathbb{E}\left[Q_{i}\left(t_{k}\right)\right]+$ $T \lambda_{i}$. Therefore, for $T_{K} \doteq K T$, we have

$$
\begin{aligned}
& \limsup _{T_{K} \rightarrow \infty} \frac{1}{K T} \sum_{t=0}^{K T-1} \sum_{i} \mathbb{E}\left[Q_{i}(t)\right] \\
& \leq \limsup _{K \rightarrow \infty} \frac{1}{T K} \sum_{k=0}^{K-1} \sum_{i} T\left(\mathbb{E}\left[Q_{i}\left(t_{k}\right)\right]+T \lambda_{i}\right) \leq \frac{\left(B+\xi \sum_{i} \lambda_{i}\right) T}{\xi} .
\end{aligned}
$$

Dividing by the total arrival rate into the system $\sum_{i} \lambda_{i}$ and applying Little's law, the average delay is upper bounded by an expression that is linear in the frame length $T$.

In the next section, we consider myopic policies that do not require the solution of an LP and that are able to stabilize the network for arrival rates within over $90 \%$ of the stability region. Simulation results in Section IV suggest that the myopic policies may in fact achieve the full stability region while providing better delay performance than the FBDC policy for most arrival rates.

\section{E. Myopic Control Policies}

We investigate the performance of simple myopic policies that make scheduling/switching decisions according to weight functions that are products of the queue lengths and the channel predictions for a small number of slots into the future. We refer to a myopic policy considering $k$ future time slots as the $k$-lookahead myopic policy. We implement these policies over frames of length $T$ time slots where during the $j$ th frame, the queue lengths at the beginning of the frame, $Q_{1}(j T)$ and $Q_{2}(j T)$, are used for weight calculations during the frame. Specifically, in the OLM policy, assuming that the server is with queue 1 at some $t \in\{j T, \ldots, j(T+1)-1\}$, the weight of queue 1 is the product of $Q_{1}(j T)$ and the summation of the current state of the channel process $C_{1}$ and the probability that $C_{1}$ will be in the ON state at $t+1$. The weight of queue 2 is calculated similarly; however, the current state of the channel process $C_{2}$ is not included in the weight since queue 2 is not available to the server in the current time slot. The detailed description of the OLM policy is given in Algorithm 3 as follows.

\section{Algorithm 3 OLM Policy}

1:Assuming that the server is currently with queue 1 and the system is at the $j$ th frame, calculate the following weights in each time slot of the current frame

$$
\begin{aligned}
& W_{1}(t)=Q_{1}(j T)\left(C_{1}(t)+\mathbb{E}\left[C_{1}(t+1) \mid C_{1}(t)\right]\right) \\
& W_{2}(t)=Q_{2}(j T) \mathbb{E}\left[C_{2}(t+1) \mid C_{2}(t)\right] .
\end{aligned}
$$

2:If $W_{1}(t) \geq W_{2}(t)$ stay with queue 1 ; otherwise, switch to the other queue. A similar rule applies for queue 2.

Next, we establish a lower bound on the stability region of the OLM policy by comparing its drift over a frame to the drift of the FBDC policy. 
TABLE III

OLM POLICY MAPPING FROM THE QUEUE SizeS TO THE CORNERS OF $\boldsymbol{\Lambda}_{\mathrm{s}}, b_{0}, b_{1}, b_{2}, b_{3}, b_{4}, b_{5}$ SHOWN IN FIG. 4(A), FOR $\epsilon<\epsilon_{c}$. FOR EACH STATE $\mathbf{s}=\left(m(t), C_{1}(t), C_{2}(t)\right)$ THE OPTIMAL ACTION IS SPECIFIED. THE THRESHOLDS ON $Q_{2} / Q_{1}$ ARE $0, T_{1}=\epsilon /(1-\epsilon), T_{2}=(1-\epsilon) /(2-\epsilon)$, $1, T_{3}=(2-\epsilon) /(1-\epsilon), T_{4}=(1-\epsilon) / \epsilon$. THE CORRESPONDING THRESHOLDS FOR THE FBDC POLICY ARE $0, T_{1}^{*}, T_{2}^{*}, 1, T_{3}^{*}, T_{4}^{*}$. FOR EXAMPLE, CORNER $b_{2}$ IS CHOSEN IN THE FBDC POLICY IF $1 \leq Q_{2} / Q_{1}<T_{3}^{*}$, WHEREAS IN THE OLM POLICY IF $1 \leq Q_{2} / Q_{1}<T_{3}$

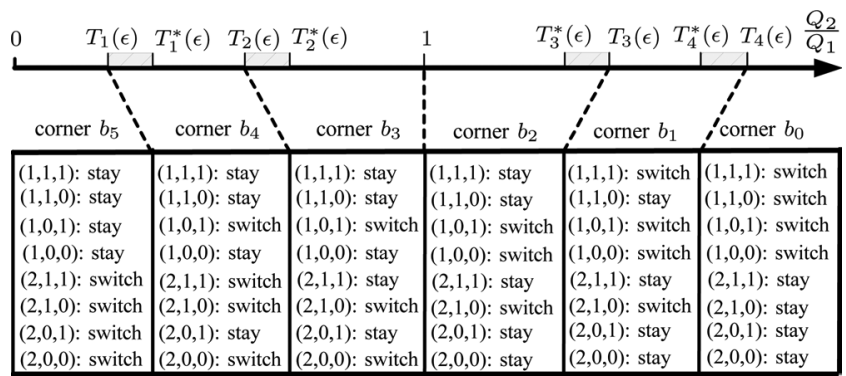

TABLE IV

OLM POLICY MAPPING From THE QUEUE Sizes TO THE CORNERS OF $\boldsymbol{\Lambda}_{\mathbf{s}}, b_{0}, b_{1}, b_{2}, b_{3}$ SHOWN IN Fig. 4(B), FOR $\epsilon \geq \epsilon_{c}$. FOR EACH STATE $\mathbf{s}=\left(m(t), C_{1}(t), C_{2}(t)\right)$, THE OPTIMAL ACTION IS SPECIFIED. THE THRESHOLDS ON $Q_{2} / Q_{1}$ ARE $0, T_{1}=\epsilon /(1-\epsilon), 1, T_{2}=(1-\epsilon) / \epsilon$. THE CORRESPONDING THRESHOLDS FOR THE FBDC POLICY ARE $0, T_{1}^{*}, 1, T_{2}^{*}$. FOR EXAMPLE, CORNER $b_{1}$ IS CHOSEN IN THE FBDC POLICY IF $1 \leq Q_{2} / Q_{1}<T_{2}^{*}$, WHEREAS IN THE OLM POLICY IF $1 \leq Q_{2} / Q_{1}<T_{2}$

\begin{tabular}{|c|c|c|c|}
\hline corner $b_{5}$ & corner $b_{3}$ & corner $b_{2}$ & corner $b_{0}$ \\
\hline $\begin{array}{l}(1,1,1): \text { stay } \\
(1,1,0): \text { stay } \\
(1,0,1): \text { stay } \\
(1,0,0): \text { stay } \\
(2,1,1): \text { switch } \\
(2,1,0): \text { switch } \\
(2,0,1): \text { switch } \\
(2,0,0): \text { switch }\end{array}$ & $\begin{array}{l}(1,1,1): \text { stay } \\
(1,1,0) \text { stay } \\
(1,0,1): \text { switch } \\
(1,0,0): \text { stay } \\
(2,1,1) \text { : stay } \\
(2,1,0) \text { : switch } \\
(2,0,1): \text { stay } \\
(2,0,0): \text { switch }\end{array}$ & $\begin{array}{l}(1,1,1): \text { stay } \\
(1,1,0) \text { : stay } \\
(1,0,1): \text { switch } \\
(1,0,0): \text { switch } \\
(2,1,1) \text { : stay } \\
(2,1,0) \text { : switch } \\
(2,0,1) \text { : stay } \\
(2,0,0) \text { : stay }\end{array}$ & $\begin{array}{l}(1,1,1): \text { switch } \\
(1,1,0): \text { switch } \\
(1,0,1): \text { switch } \\
(1,0,0): \text { switch } \\
(2,1,1) \text { : stay } \\
(2,1,0) \text { : stay } \\
(2,0,1): \text { stay } \\
(2,0,0) \text { : stay }\end{array}$ \\
\hline
\end{tabular}

Theorem 5: The OLM policy achieves at least $\gamma$-fraction of the stability region $\Lambda_{\mathrm{s}}$ asymptotically in $T$ where $\gamma \geq 90 \%$.

The proof is constructive and it is establish in various steps in Appendix D. The basic idea behind the proof is that the OLM policy produces mappings from the set of queue sizes to the stationary-deterministic policies corresponding to the corners of the stability region. These mappings are similar to those of the FBDC policy; however, the thresholds on the queue size ratios $Q_{2} / Q_{1}$ are determined according to (13). Tables III and IV show these mappings for $\epsilon<\epsilon_{c}$ and $\epsilon \geq \epsilon_{c}$, respectively. These mappings are in general different from the corresponding mappings of the FBDC policy in Tables I and II.

Therefore, for a given ratio of the queue sizes $Q_{2} / Q_{1}$, the FBDC and the OLM policies may apply different stationary-deterministic policies corresponding to different corner points of $\boldsymbol{\Lambda}_{\mathbf{s}}$, denoted by $\mathbf{r}^{*}$ and $\hat{\mathbf{r}}$, respectively. The shaded intervals of $Q_{2} / Q_{1}$ in Tables III and IV are the intervals in which the OLM and the FBDC policies apply different policies. The corresponding mapping for the OLM policy for the case of nonsymmetric Gilbert-Elliot channels is given in Appendix B. The proof of Theorem 5 is then completed by first establishing the bound on the weighted average departure rate of the OLM policy with respect to that of the FBDC policy in (14), and then showing that this is a sufficient condition for the OLM policy to achieve at least $90 \%$ of $\boldsymbol{\Lambda}_{\mathrm{s}}$ asymptotically in $T$

$$
\Psi \doteq \frac{\sum_{i} Q_{i}(t) \hat{r}_{i}}{\sum_{i} Q_{i}(t) r_{i}^{*}} \geq 90 \%
$$

A similar analysis shows that the 2-lookahead myopic policy achieves at least $94 \%$ of $\boldsymbol{\Lambda}_{s}$, while the 3-lookahead myopic policy achieves at least $96 \%$ of $\boldsymbol{\Lambda}_{s}$. The $k$-lookahead myopic policy is the same as earlier except that the following weight functions are used for scheduling decisions: Assuming the server is with queue 1 at time slot $t$, $W_{1}(t)=Q_{1}(j T)\left(C_{1}(t)+\sum_{\tau=1}^{k} \mathbb{E}\left[C_{1}(t+\tau) \mid C_{1}(t)\right]\right)$ and $W_{2}(t)=Q_{2}(j T) \sum_{\tau=1}^{k} \mathbb{E}\left[C_{2}(t+\tau) \mid C_{2}(t)\right]$.

\section{GENERAL SYSTEM}

In this section, we extend the results developed in the previous section to the general case of an arbitrary number of queues in the system.

\section{A. Model}

Consider the same model as in Section II-A with $N>1$ queues for some $N \in \mathbb{N}$ as shown in Fig. 1. Let the i.i.d. process $A_{i}(t)$ with arrival rate $\lambda_{i}$ denote the number of arrivals to queue $i$ at time slot $t$, where $\mathbb{E}\left[A_{i}^{2}(t)\right] \leq A_{\max }^{2}, i \in\{1,2, \ldots, N\}$. Let $C_{i}(t)$ be the channel (connectivity) process of queue $i, i \in$ $\{1,2, \ldots, N\}$, that forms the two-state Markov chain with transition probabilities $p_{01}$ and $p_{10}$ as shown in Fig. 2. We assume that the processes $A_{i}(t), i \in\{1, \ldots, N\}$ and $C_{i}(t), i \in$ $\{1, \ldots, N\}$ are independent. It takes one slot for the server to switch from one queue to the other, and $m(t) \in\{1, \ldots, N\}$ denotes the queue at which the server is present at slot $t$. Let $\mathbf{s}_{t}=\left(m(t), C_{1}(t), \ldots, C_{N}(t)\right) \in \mathcal{S}$ denote the state of the corresponding saturated system at time $t$ where $\mathcal{S}$ is the set of all states. The action $a(t)$ in each time slot is to choose the queue at which the server will be present in the next time slot, i.e., $a_{t} \in\{1, \ldots, N\} \doteq \mathcal{A}$ where $\mathcal{A}$ is the set of all actions at each state.

\section{B. Stability Region}

In this section, we characterize the stability region of the general system under nonsymmetric channel models. ${ }^{3}$ For the case of i.i.d. channel processes, we explicitly characterize the stability region and the throughput-optimal policy. For Markovian channel models, we extend the stability region characterization in terms of state-action frequencies to the general system. Furthermore, we develop a tight outer bound on the stability region using an upper bound on the sum throughput and show that a simple myopic policy achieves this upper bound for the corresponding saturated system.

A dynamic server allocation problem over parallel channels with randomly varying connectivity and limited channel sensing has been investigated in [1], [2] and [45] under the Gilbert-Elliot channel model. The goal in [1], [2] and [45] is to maximize the sum rate for the saturated system, where it is proved that a

${ }^{3}$ For Markovian (Gilbert-Elliot) channels, we preserve the symmetry of the channel processes across the queues. 
myopic policy is optimal. In this section, we prove that a myopic policy is sum-rate optimal under the Gilbert-Elliot channel model and one-slot server switchover delay. Furthermore, our goal is to characterize the set of all achievable rates, i.e., the stability region, together with a throughput-optimal scheduling algorithm for the dynamic queuing system.

Memoryless Channels: The results established in Section II-B for the case of i.i.d. connectivity processes can easily be extended to the system of $N$ queues with nonsymmetric i.i.d. channels as the same intuition applies for the general case. We state this result in the following theorem whose proof can be found in [14].

Theorem 6: For a system of $N$ queues with arbitrary switchover delays and i.i.d. channels with probabilities $p_{i}, i \in\{1, \ldots, N\}$, the stability region $\Lambda$ is given by

$$
\boldsymbol{\Lambda}=\left\{\lambda \geq \mathbf{0} \mid \sum_{i=1}^{N} \frac{\lambda_{i}}{p_{i}} \leq 1\right\} .
$$

In addition, the simple exhaustive (gated) policy is throughput optimal.

As for the case of two queues, the simultaneous presence of randomly varying connectivity and the switchover delay significantly reduces the stability region as compared to the corresponding system without switchover delay analyzed in [39]. Furthermore, when the channel processes are memoryless, no policy can take advantage of the channel diversity as the simple queue-blind exhaustive-type policies are throughput optimal.

In the next section, we show that, similar to the case of two queues, the memory in the channel improves the stability region of the general system.

Channels With Memory: Similar to Section II-C, we start by establishing the rate region $\boldsymbol{\Lambda}_{s}$ by formulating an MDP for rate maximization in the corresponding saturated system. The reward functions in this case are given as follows:

$$
\bar{r}_{i}(\mathbf{s}, a) \doteq 1 \text { if } m=i, C_{i}=1 \text {, and } a=i, i=1, \ldots, N
$$

and $\bar{r}_{i}(\mathbf{s}, a) \doteq 0$ otherwise, where $m$ denotes the queue at which the server is present. That is, one reward is obtained when the server stays at a queue with an ON channel. Given some $\alpha_{i} \geq$ $0, i \in\{1, \ldots, N\}, \sum_{i} \alpha_{i}=1$, we define the system reward at time $t$ as

$$
\bar{r}(\mathbf{s}, a) \doteq \sum_{i=1}^{N} \alpha_{i} \bar{r}_{i}(\mathbf{s}, a)
$$

The average reward of policy $\pi$ is defined as

$$
r^{\pi} \doteq \lim _{K \rightarrow \infty} \frac{1}{K} E\left\{\sum_{t=1}^{K} \bar{r}\left(\mathbf{s}_{t}, a_{t}^{\pi}\right)\right\}
$$

Therefore, the problem of maximizing the time-average expected reward over all policies, $r^{*} \doteq \max _{\pi} r^{\pi}$, is a discrete time MDP characterized by the state transition probabilities $\mathbf{P}\left(\mathbf{s}^{\prime} \mid \mathbf{s}, a\right)$ with $N 2^{N}$ states and $N$ possible actions per state. Furthermore, similar to the two-queue system, there exists a positive probability path between any given pair of states under some stationary-deterministic policy. Therefore, this MDP belongs to the class of weakly communicating MDPs [34] for which there exists a stationary-deterministic optimal policy independent of the initial state [34]. The state-action polytope, $\mathbf{X}$ is the set of state-action frequency vectors $\mathbf{x}$ that satisfy the balance equations

$$
\sum_{a \in \mathcal{A}} \mathbf{x}(\mathbf{s}, a)=\sum_{\mathbf{s}^{\prime} \in \mathcal{S}} \sum_{a \in \mathcal{A}} \mathbf{P}\left(\mathbf{s} \mid \mathbf{s}^{\prime}, a\right) \mathbf{x}\left(\mathbf{s}^{\prime}, a\right), \forall \mathbf{s} \in \mathcal{S}
$$

the normalization condition

$$
\sum_{\mathbf{s} \in \mathcal{S}} \sum_{a \in \mathcal{A}} \mathbf{x}(\mathbf{s}, a)=1
$$

and the nonnegativity constraints

$$
\mathbf{x}(\mathbf{s}, a) \geq 0, \text { for } \mathbf{s} \in \mathcal{S}, a \in \mathcal{A}
$$

where the transition probabilities $\mathbf{P}\left(\mathbf{s} \mid \mathbf{s}^{\prime}, a\right)$ are functions of the channel parameters $p_{10}$ and $p_{01}$. The following linear transformation of the state-action polytope $\mathbf{X}$ defines the rate polytope $\boldsymbol{\Lambda}_{s}$, namely, the set of all time-average expected rate pairs that can be obtained in the saturated system

$$
\boldsymbol{\Lambda}_{s}=\left\{\mathbf{r} \mid r_{i}=\sum_{\mathbf{s} \in \mathcal{S}} \sum_{a \in \mathcal{A}} \mathbf{x}(\mathbf{s}, a) \bar{r}_{i}(\mathbf{s}, a), \mathbf{x} \in \mathbf{X}, i \in\{1,2, \ldots, N\}\right\}
$$

where the reward functions $\bar{r}_{i}(\mathbf{s}, a), i \in\{1, \ldots, N\}$, are defined in (15). Algorithm 4 gives an alternative characterization of the rate region $\boldsymbol{\Lambda}_{s}$.

Algorithm 4 Stability Region Characterization

1:Given $\alpha_{1}, \ldots, \alpha_{N} \geq 0, \sum_{i} \alpha_{i}=1$, solve the following LP

$$
\begin{aligned}
& \max _{\mathbf{x}} . \sum_{i=1}^{N} \alpha_{i} r_{i}(\mathbf{x}) \\
& \text { subject to } \mathbf{x} \in \mathbf{X} .
\end{aligned}
$$

2:There exists an optimal solution $\left(r_{1}^{*}, \ldots, r_{N}^{*}\right)$ of this LP that lies at a corner point of $\boldsymbol{\Lambda}_{\mathrm{s}}$. Find all possible corner points and take their convex combination.

Similar to the two-queue case, the fundamental theorem of linear programming guarantees existence of an optimal solution to (17) at a corner point of the polytope $\mathbf{X}$ [8]. We will establish in the next section that the rate region $\boldsymbol{\Lambda}_{s}$ is in fact achievable in the dynamic queuing system, which will imply that $\boldsymbol{\Lambda}=\boldsymbol{\Lambda}_{s}$. For the case of three queues, Fig. 5 shows the stability region $\Lambda$. As expected, the stability region is significantly reduced as compared to the corresponding system with zero switchover delays analyzed in [39].

Analytical Outer Bound for the Stability Region: In this section, we first derive an upper bound to the sum throughput in the saturated system and then use it to characterize an outer bound to the rate region $\boldsymbol{\Lambda}_{s}$. Let $C_{0}^{(N)} \doteq \frac{p_{10}^{N}}{\left(p_{10}+p_{01}\right)^{N}}$ denote the probability that all channels are in OFF state in steady state. 


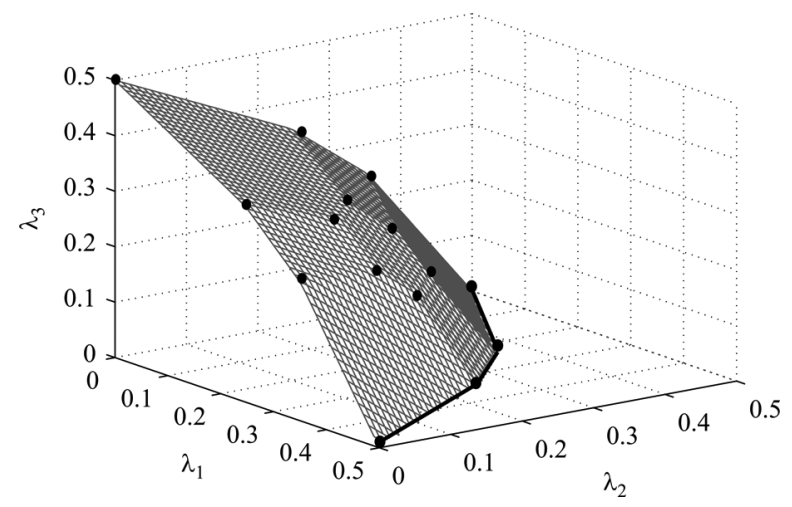

Fig. 5. Stability region for three parallel queues for $p_{10}=p_{01}=0.3$.

Lemma 2: An upper bound on the sum rate in the saturated system is given by

$$
\sum_{i=1}^{N} r_{i} \leq 1-C_{0}^{(N)}-\left(p_{10}\left(1-C_{0}^{(N)}\right)-p_{01} C_{0}^{(N)}\right) .
$$

The proof is given in Appendix E. In the next section, we propose a simple myopic policy for the saturated system that achieves this upper bound. Similar to the case of two queues, the surface $\sum_{i=1}^{N} r_{i} \leq 1-C_{0}^{(N)}$ is one of the boundaries of the stability region for the system without switchover delay analyzed in [39], where the probability that at least 1 channel is in ON state in steady state is $1-C_{0}^{(N)}$. Therefore, $p_{10}\left(1-C_{0}^{(N)}\right)-p_{01} C_{0}^{(N)}$ is the throughput loss due to one slot switchover delay in our system. The analysis of the myopic policy in the next section shows that this throughput loss due to switchover delay corresponds to the probability that the server is at a queue with OFF state when at least one other queue is in ON state. For the case of $N=3$ queues, the sum throughput upper bound in Lemma 2 is the hexagonal region at the center of the plot in Fig. 5.

Because any convex combination of $r_{i}, i \in\{1, \ldots, N\}$, must lie under the sum-rate surface, (18) is in fact an outer bound on the whole rate region $\boldsymbol{\Lambda}_{s}$. Furthermore, no queue can achieve a time-average expected rate that is greater than the steady-state probability that the corresponding queue is in ON state, i.e., $p_{01} /\left(p_{10}+p_{01}\right)$. Therefore, the intersection of these $N+1$ surfaces in the $N$-dimensional space constitutes an outer bound for the rate region $\boldsymbol{\Lambda}_{s}$. Note that this outer bound is tight in that the sum-rate surface of the maximum rate region $\boldsymbol{\Lambda}_{s}$ as well as the corner points $p_{01} /\left(p_{10}+p_{01}\right)$ coincide with the outer bound. This outer bound with respect to the rate region are displayed in Fig. 6 for the case of $N=3$ nodes.

\section{Myopic Policy for the Saturated System}

We show in this section that a simple and intuitive policy, termed the greedy myopic (GM) policy, achieves the sum-rate maximization for the saturated system. This policy is a greedy policy in that under the policy, if the current queue is available to serve, the server serves it. Otherwise, the server switches to a queue with $\mathrm{ON}$ channel state, if such a queue exists. The policy is described in Algorithm 5. Recall that $m(t)$ denotes the queue the server is present at time slot $t$.

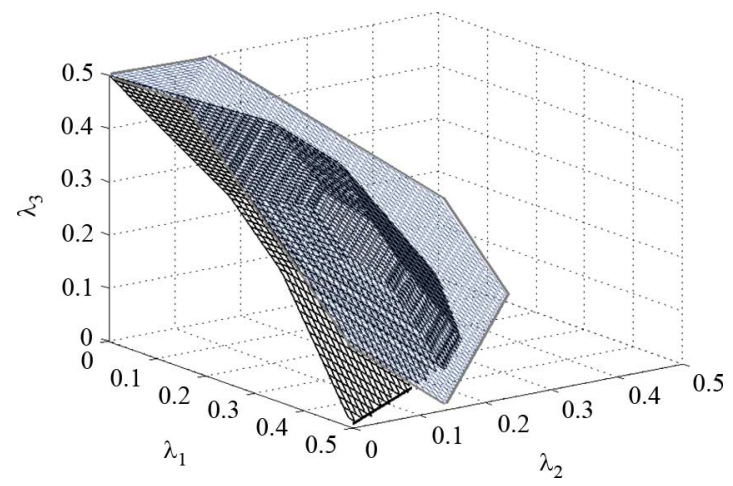

Fig. 6. Stability region outer bound for three parallel queues for $p_{10}=p_{01}=$ 0.3 .

\section{Algorithm 5 GM Policy}

1:For all time slots $t$, if $C_{m(t)}(t)=1$, serve queue $m(t)$.

2:Otherwise, if $\exists j \in\{1, \ldots, N\}, j \neq m(t)$, such that $C_{j}(t)=1$, among the queues that have ON channel state, switch to the queue with the smallest index in a cyclic order starting from queue $m(t)$.

The cyclic switching order under the GM policy is as follows: If the server is at queue $i$ and the decision is to switch, then the server switches to queue $j$, where for $i=N, j=\arg \min _{j \in\{1, \ldots, N-1\}}\left(C_{j}(t)=1\right)$ and for $i \neq N$ if $\exists j \in\{i+1, \ldots, N\}$, such that $C_{j}(t)=1$, we have $j=\arg \min _{j \in\{i+1, \ldots, N\}}\left(C_{j}(t)=1\right)$, if not, then $j=\arg \min _{j \in\{1, \ldots, i-1\}}\left(C_{j}(t)=1\right)$.

Theorem 7: The GM policy achieves the sum-rate upper bound.

Proof: Given a fixed decision rule at each state, the system state forms a finite state space, irreducible and positive recurrent Markov chain. Therefore, under the GM policy, the system state converges to a steady-state distribution. We partition the total probability space into three disjoint events.

$E_{1}$ : the event that all the channels are in OFF state.

$E_{2}$ : the event that at least one channel is in ON state and the server is at a queue with ON state.

$E_{3}$ : the event that at least one channel is in ON state and the server is at a queue with OFF state.

Since these events are disjoint and cover the whole sample space, we have

$$
1=\mathbb{P}\left(E_{1}\right)+\mathbb{P}\left(E_{2}\right)+\mathbb{P}\left(E_{3}\right) .
$$

We have $\mathbb{P}\left(E_{1}\right)=C_{0}^{(N)}$ by definition. Since the GM policy decides to serve the current queue if it is in ON state, $\mathbb{P}\left(E_{2}\right)$ gives the sum throughput $\sum_{i} r_{i}$ for the GM policy. Therefore, we have that under the GM policy

$$
\sum_{i} r_{i}=1-C_{0}^{(N)}-\mathbb{P}\left(E_{3}\right)
$$

We show that $\mathbb{P}\left(E_{3}\right)=p_{10}\left(1-C_{0}^{(N)}\right)-p_{01} C_{0}^{(N)}$. Consider a time slot $t$ in steady state and let $\kappa(t)$ be the number of channels 
with ON state at time slot $t$ and let $E_{0}(t)$ be the event that the server is at a queue with OFF state at time slot $t$. We have

$$
\begin{aligned}
\mathbb{P}\left(E_{3}\right) & =\mathbb{P}\left(E_{0}(t) \text { and } 1 \leq \kappa(t) \leq N-1\right)=\mathbb{P}\left(E_{0}(t) \text { and } \kappa(t) \geq 1\right) \\
& =\mathbb{P}\left(E_{0}(t) \text { and } \kappa(t) \geq 1 \mid \kappa(t-1) \geq 1\right) \mathbb{P}(\kappa(t-1) \geq 1) \\
& +\mathbb{P}\left(E_{0}(t) \text { and } \kappa(t) \geq 1 \mid \kappa(t-1)=0\right) \mathbb{P}(\kappa(t-1)=0)
\end{aligned}
$$

Since $t$ is a time slot in steady state, we have that $\mathbb{P}(\kappa(t-1)=$ $0)=C_{0}^{(N)}$. Therefore, $\mathbb{P}\left(E_{3}\right)$ is given by

$$
\begin{aligned}
& \mathbb{P}\left(\kappa(t) \geq 1 \mid E_{0}(t), \kappa(t-1) \geq 1\right) \mathbb{P}\left(E_{0}(t) \mid \kappa(t-1) \geq 1\right)\left(1-C_{0}^{(N)}\right) \\
& +\mathbb{P}\left(\kappa(t) \geq 1 \mid E_{0}(t), \kappa(t-1)=0\right) \mathbb{P}\left(E_{0}(t) \mid \kappa(t-1)=0\right) C_{0}^{(N)} .
\end{aligned}
$$

We have $\mathbb{P}\left(E_{0}(t) \mid \kappa(t-1) \geq 1\right)=p_{10}$ because the GM policy chooses a queue with ON state if there is such a queue. Similarly, $\mathbb{P}\left(E_{0}(t) \mid \kappa(t-1)=0\right)$ is the probability that the queue chosen by the GM policy keeps its OFF channel state, given by $1-p_{01}$

$$
\begin{aligned}
\mathbb{P}\left(E_{3}\right) & =\left(1-\mathbb{P}\left(\kappa(t)=0 \mid E_{0}(t), \kappa(t-1) \geq 1\right)\right) p_{10}\left(1-C_{0}^{(N)}\right) \\
& +\left(1-\mathbb{P}\left(\kappa(t)=0 \mid E_{0}(t), \kappa(t-1)=0\right)\right)\left(1-p_{01}\right) C_{0}^{(N)} \\
& =p_{10}\left(1-C_{0}^{(N)}\right)\left(1-\frac{\mathbb{P}\left(\kappa(t)=0, E_{0}(t) \mid \kappa(t-1) \geq 1\right)}{\mathbb{P}\left(E_{0}(t) \mid \kappa(t-1) \geq 1\right)}\right) \\
& +\left(1-p_{01}\right) C_{0}^{(N)}\left(1-\frac{\mathbb{P}\left(\kappa(t)=0, E_{0}(t) \mid \kappa(t-1)=0\right)}{\mathbb{P}\left(E_{0}(t) \mid \kappa(t-1)=0\right)}\right) \\
& =p_{10}\left(1-C_{0}^{(N)}\right)\left(1-\frac{\mathbb{P}(\kappa(t)=0 \mid \kappa(t-1) \geq 1)}{p_{10}}\right) \\
& +\left(1-p_{01}\right) C_{0}^{(N)}\left(1-\frac{\mathbb{P}(\kappa(t)=0 \mid \kappa(t-1)=0)}{1-p_{01}}\right) .
\end{aligned}
$$

Using Bayes' rule on the event $\{\kappa(t)=0\}$, we have that $\mathbb{P}(\kappa(t)=0 \mid \kappa(t-1) \geq 1)$ is given by

$$
\frac{\mathbb{P}(\kappa(t)=0)-\mathbb{P}(\kappa(t)=0 \mid \kappa(t-1)=0) \mathbb{P}(\kappa(t-1)=0)}{\mathbb{P}(\kappa(t-1) \geq 1)}
$$

which is equivalent to $\left(C_{0}^{(N)}-\left(1-p_{01}\right)^{N} C_{0}^{(N)}\right) /\left(1-C_{0}^{(N)}\right)$. Therefore, $\mathbb{P}\left(E_{3}\right)$ is given by

$$
\begin{aligned}
\mathbb{P}\left(E_{3}\right) & =p_{10}\left(1-C_{0}^{(N)}\right)\left(1-\frac{C_{0}^{(N)}-\left(1-p_{01}\right)^{N} C_{0}^{(N)}}{p_{10}\left(1-C_{0}^{(N)}\right)}\right) \\
& +\left(1-p_{01}\right) C_{0}^{(N)}\left(1-\frac{\left(1-p_{01}\right)^{N}}{1-p_{01}}\right) \\
& =p_{10}\left(1-C_{0}^{(N)}\right)-p_{01} C_{0}^{(N)} .
\end{aligned}
$$

As mentioned in the previous section, $\mathbb{P}\left(E_{3}\right)$ is the throughput loss due to switching as it represents the fraction of time the server is at a queue with OFF state when there are queues with ON state in the system.

\section{FBDC Policy}

In this section, we generalize the FBDC policy to the general system and show that it is throughput optimal asymptoti- cally in the frame length for the general case. The FBDC algorithm for the general system is very similar to the FBDC algorithm described for two queues in Section II-D. Specifically, the time is divided into equal-size intervals of $T$ slots. We find the stationary-deterministic policy that optimally solves (17) for the saturated system when $Q_{1}(j T), \ldots, Q_{N}(j T)$ are used as weights and then apply this policy in each time slot of the frame in the actual system. The FBDC policy is described in Algorithm 6 in details.

\section{Algorithm 6 FBDC Policy}

1:Find the optimal solution to the following LP:

$$
\begin{aligned}
\max \cdot\{\mathbf{r}\} & \sum_{i=1}^{N} Q_{i}(j T) r_{i} \\
\text { subject to } & \mathbf{r}=\left(r_{1}, \ldots, r_{N}\right) \in \boldsymbol{\Lambda}_{s}
\end{aligned}
$$

where $\Lambda_{s}$ is the rate region for the saturated system.

2:The optimal solution $\left(r_{1}^{*}, \ldots, r_{N}^{*}\right)$ in step 1 is a corner point of $\boldsymbol{\Lambda}_{s}$ that corresponds to a stationary-deterministic policy denoted by $\pi^{*}$. Apply $\pi^{*}$ in each time slot of the frame.

Theorem 8: For any $\delta>0$, there exists a large enough frame length $T$ such that the FBDC policy stabilizes the system for all arrival rates within the $\delta$-stripped stability region $\Lambda_{s}^{\delta}=\Lambda_{s}-\delta \mathbf{1}$.

The proof is very similar to the proof of Theorem 4 and it can be found in [14]. The theorem establishes the asymptotic throughput optimality of the FBDC policy for the general system.

Remark 3: The FBDC policy provides a new framework for developing throughput-optimal policies for network control. Namely, given any queuing system whose corresponding saturated system is Markovian with finite state and action spaces, throughput optimality is achieved by solving an LP in order to find the stationary MDP solution of the corresponding saturated system and applying this solution over a frame in the actual system. In particular, the FBDC policy can stabilize systems with arbitrary switchover times and more complicated Markov modulated channel structures. The FBDC policy can also be used to achieve throughput optimality for classical network control problems such as the parallel queuing systems in [32] and [39], scheduling in switches in [35], or scheduling under delayed channel state information [44].

Similar to the delay analysis in Section II-D1 for the twoqueue system, a delay upper bound that is linear in the frame length $T$ can be obtained for the FBDC policy for the general system. Moreover, the FBDC policy for the general system can also be implemented without any frames by setting $T=1$, i.e., by solving the LP in Algorithm 6 in each time slot. The simulation results regarding such implementations suggest that the FBDC policy implemented without frames has a similar throughput performance and an improved delay performance as compared to the original FBDC policy.

Discussion: For systems with switchover delay, it is well known that the celebrated MW scheduling policy is not 
throughput optimal [11]. In the absence of randomly varying connectivity, variable frame-based generalizations of the MW policy are throughput optimal [15]. However, when the switchover delay and randomly varying connectivity are simultaneously present in the system, the FBDC policy is the only policy to achieve throughput optimality and it has a significantly different structure from the MW policy.

The FBDC policy for a fixed frame length $T$ does not require the arrival rate information for stabilizing the system for arrival rates in $\boldsymbol{\Lambda}-\delta(T) \mathbf{1}$; however, it requires the knowledge of the channel connectivity parameters $p_{10}$ and $p_{01}$. To deal with this problem, one can estimate the channel parameters periodically and use these estimates to solve the LP in (19). This approach, of course, incurs a throughput loss depending on how large the estimation error is.

As mentioned in Remark 3, the FBDC policy can stabilize a large class of network control problems whose corresponding saturated system is weakly communicating Markovian with a finite state and action spaces. However, one caveat of the FBDC policy is that the state space of the LP that needs to be solved increases exponentially with the number of links in the system. The celebrated MW policy (which is not stabilizing for the system considered here) has linear complexity for the single server system considered in this paper. However, for a general multiserver system or a single hop network with $N$ interfering links, the MW policy has to solve a maximum-independent set problem over all links at each time slot, which is a hard problem whose state space is exponential in the number of links $N$. The FBDC policy on the other hand, only has to solve an LP, for which there are standard solvers available such as CPLEX. Furthermore, the FBDC policy has to solve the LP once per frame, whereas the MW policy performs maximum-independent set computation each time slot. If the frame length for the FBDC policy is chosen to be bigger than the computational complexity of the LP in (19), then the per-slot computational complexity of the algorithm is reduced to $O(1)$ [33]. Such a frame-based implementation is also possible for the MW policy to reduce its complexity to $O(1)$ per time slot [33]. On the other hand, the shortcoming of such an approach for both policies is the increase in delay as a result of the larger frame length. This outlines a tradeoff between complexity and delay, whereby a reduction in complexity by adjusting the frame length comes at the expense of delay.

The MW policy was first introduced in [38] for multihop networks and despite its exponential complexity in number of links, it provided a useful structure for designing queue lengthbased scheduling algorithms. Later, the structure suggested by the MW policy lead to suboptimal but low-complexity algorithms, as well as distributed implementations of the policy for certain systems (see, e.g., greedy-maximal network scheduling in [43]). Our aim in proposing the FBDC policy and the state-action frequency framework for network scheduling is to give a structure for throughput-optimal algorithms for systems with time-varying channels and switchover delays, and hopefully to provide insight into designing scalable algorithms that can stabilize such systems. The myopic control policies we discuss in the next section constitute a first attempt toward characterizing the structure of more scalable algorithms.

\section{E. Myopic Control Policies}

In this section, we generalize myopic policies that we introduced for the two-queue system in Section II to the general system. Myopic policies make scheduling decisions based on queue lengths and simple channel predictions into the future. We present an implementation of these policies over frames of length $T$ time slots where during the $j$ th frame, the queue lengths at the beginning of the frame, $Q_{1}(j T), \ldots, Q_{N}(j T)$, are used for weight calculations during the frame. We describe the OLM policy for the general system in Algorithm 7.

\section{Algorithm 7 OLM Policy}

1:Assuming that the server is currently with queue 1 and the system is at the $j$ th frame, calculate the following weights in each time slot of the current frame

$$
\begin{aligned}
& W_{1}(t)=Q_{1}(j T)\left(C_{1}(t)+\mathbb{E}\left[C_{1}(t+1) \mid C_{1}(t)\right]\right) \\
& W_{i}(t)=Q_{i}(j T) \mathbb{E}\left[C_{i}(t+1) \mid C_{i}(t)\right], \quad i \neq 1 .
\end{aligned}
$$

2:If $W_{1}(t) \geq W_{i}(t), \forall i \in\{2, \ldots, N\}$, then stay with queue 1 . Otherwise, switch to a queue that achieves

$$
\max _{i} Q_{i}(j T) \mathbb{E}\left[C_{i}(t+1) \mid C_{i}(t)\right] .
$$

A similar rule applies when the server is at other queues.

The technique used for the case of two queues for analyzing the stability region achieved by the OLM policy is extremely cumbersome to generalize to the system with $N$ queues. Therefore, for the general system, we have investigated the performance of the OLM policy in simulations. The simulation results in Section IV suggest that the OLM policy may achieve the full stability region while providing a better delay performance as compared to the FBDC policy.

Similar to the FBDC policy, the myopic policies can be implemented without the use of frames by setting $T=1$, i.e., by utilizing the current queue lengths for updating the decision rules every time slot. This could potentially lead to more delay-efficient policies that are more adaptive to dynamic changes in queue lengths. We elaborate on this through the numerical results in the next section.

Similar to the system with two queues, the $k$-lookahead myopic policy is the same as earlier except that the following weight functions are used for scheduling decisions: Assuming the server is with queue 1 at time slot $t$, $W_{1}(t)=Q_{1}(j T)\left(C_{1}(t)+\sum_{\tau=1}^{k} \mathbb{E}\left[C_{1}(t+\tau) \mid C_{1}(t)\right]\right)$ and $W_{i}(t)=Q_{i}(j T) \sum_{\tau=1}^{k} \mathbb{E}\left[C_{i}(t+\tau) \mid C_{i}(t)\right], i \in\{2, \ldots, N\}$. These policies have very low complexity and they are simpler to implement as compared to the FBDC policy.

\section{NUMERICAL RESULTS}

We performed simulation experiments that present average queue occupancy results for the FBDC, the OLM, and the MW policies for systems with $N=2$ or $N=3$ queues. We first verified that in the simulation results for the FBDC policy, queue sizes grow unbounded only for arrival rates outside the stability region, and then performed experiments for the OLM policy. In 


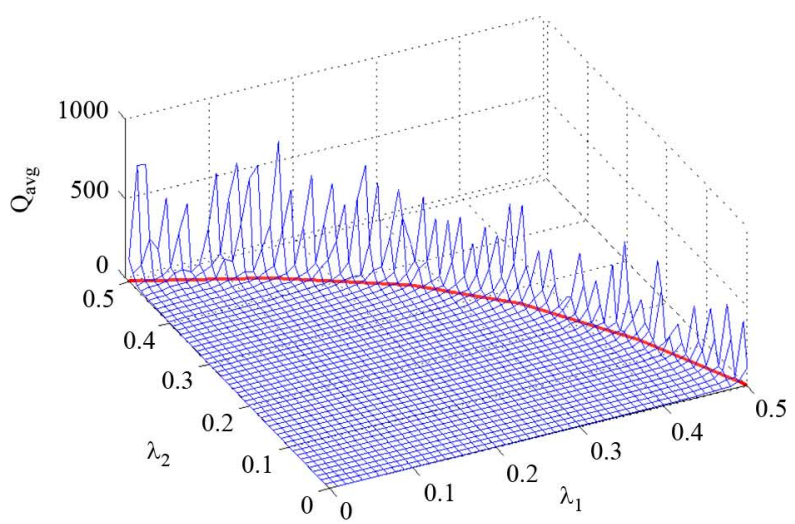

(a)

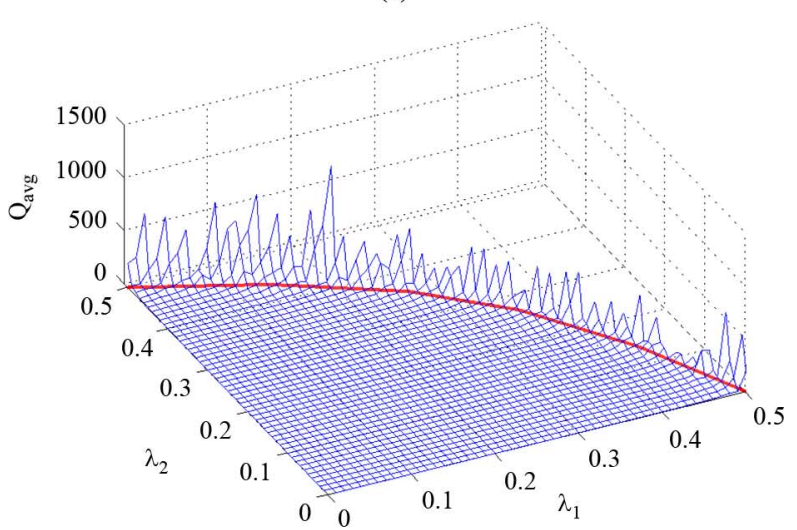

(b)

Fig. 7. Total average queue size for (a) the FBDC policy and (b) the myopic policy for $T=25$ and $\epsilon=0.25$.

all the reported results, we have $\lambda \in \Lambda$ with 0.01 increments. For each point at the boundary of $\boldsymbol{\Lambda}$, we simulated one point outside the stability region. Furthermore, for each data point, the arrival processes were i.i.d., the channel processes were Markovian as in Fig. 2, and the simulation length was $T_{s}=100000$ slots.

Fig. 7(a) presents the total average queue size, $\mathbf{Q}_{\text {avg }} \doteq$ $\sum_{t=1}^{T_{s}}\left(Q_{1}(t)+Q_{2}(t)\right) / T_{s}$, under the FBDC policy for $N=2$ queues, $\epsilon=0.25<\epsilon_{c}$, and a frame size of $T=25$ slots. The boundary of the stability region is shown by (red) lines on the 2-D $\lambda_{1}-\lambda_{2}$ plane. We observe that the average queue sizes are small for all $\left(\lambda_{1}, \lambda_{2}\right) \in \Lambda_{s}$ and the big jumps in queue sizes occur for points outside $\boldsymbol{\Lambda}$. Fig. 7(b) presents the performance of the OLM policy with $T=25$ slot frames for the same system. The simulation results suggest that there is no appreciable difference between the stability regions of the FBDC and the OLM policies. Note that the total average queue size is proportional to long-run packet-average delay in the system through Little's law. For these two figures, the average delay under the OLM policy is less than that under the FBDC policy for $81 \%$ of all arrival rates considered.

Next, we implemented the FBDC and the OLM policies without the use of any frames (i.e., for $T=1$ ). When there are no frames, the FBDC policy solves the LP in Algorithm 2 in each time slot, and the OLM policy utilizes the queue length information in the current time slot for the weight calculations in (13). Fig. 8(a) and 8(b) presents the total average queue size

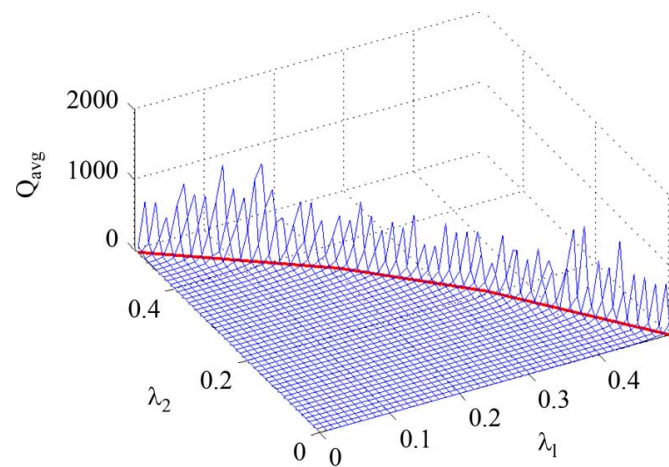

(a)

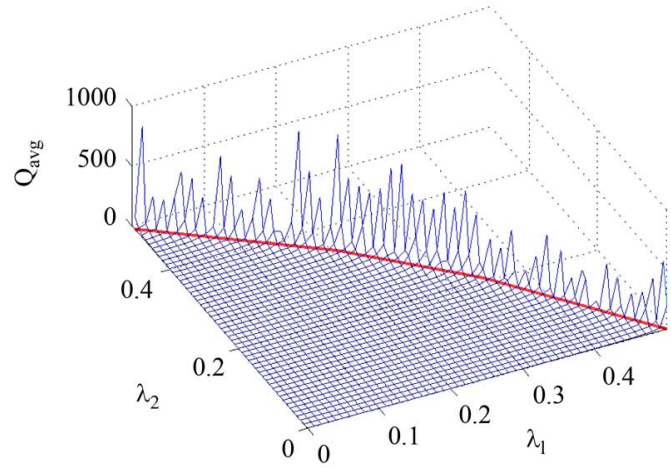

(b)

Fig. 8. Total average queue size for (a) the FBDC policy and (b) the myopic policy implemented without the use of frames (i.e., for $T=1$ ) and $\epsilon=0.40$.

under the FBDC and the OLM policies for $N=2$ queues, $T=1$, and $\epsilon=0.40>\epsilon_{c}$. Similar to the frame-based implementations, we observe that the average queue sizes are small for all $\left(\lambda_{1}, \lambda_{2}\right) \in \boldsymbol{\Lambda}$ for both policies and the big jumps in queue sizes occur for points outside $\Lambda$, which suggests that the nonframe-based implementation of the FBDC and the OLM policies may achieve the full stability region. The reason the FBDC and the OLM policies provide stability without the use of frames is that for large queue lengths, the corner point that these policies choose to apply depends completely on the queue length ratios, and hence, the choice of corner points and the associated saturated-system policies utilized in the FBDC and the OLM policies do not change fast when the queue lengths get large. Furthermore, for smaller queue lengths, the no-frame implementations of these policies are more adaptive to dynamic changes in the queue sizes as compared to implementations with large frames.

For the same system (i.e., $N=2$ queues and $\epsilon=0.40>\epsilon_{c}$ ), Fig. 9 presents the long-run packet average delay as a function of the sum throughput $\lambda_{1}+\lambda_{2}$ along the main diagonal line (i.e., $\lambda_{1}=\lambda_{2}$ ). We compare the delay performance of the FBDC and the OLM policies with $T=1$, and the MW policy which, in each time slot $t$, chooses the queue that achieves $\max _{i} Q_{i}(t) C_{i}(t)$. The maximum sum throughput is $0.75-\epsilon / 2=0.55$ as suggested by Theorem 3. Fig. 9 shows that while FBDC and the OLM policies stabilize the system for all $\lambda_{1}+\lambda_{2}<0.55$, the system becomes unstable under the MW policy around $\lambda_{1}+\lambda_{2}=0.45$. This result also confirms that the OLM policy has a much better delay performance than 


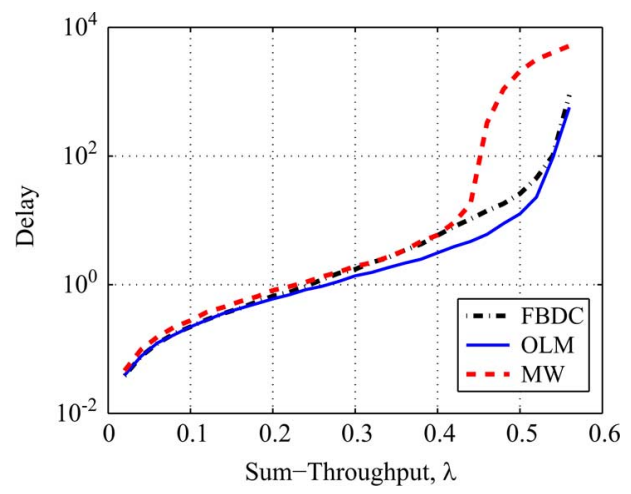

Fig. 9. Delay versus sum throughput for the FBDC, the OLM, and the MW policies implemented without the use of frames (i.e., for $T=1$ ) for $N=2$ queues and $\epsilon=0.40$.

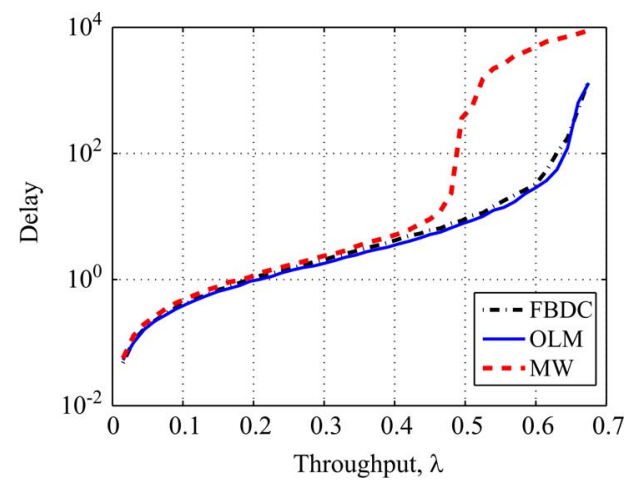

Fig. 10. Delay versus sum throughput for the FBDC, the OLM, and the MW policies implemented without the use of frames (i.e., for $T=1$ ) for $N=3$ queues and $\epsilon=0.30$.

the FBDC and the MW policies. Finally, we observed that for some small arrival rates for which the system can be stabilized under the MW policy, the FBDC and the myopic policies implemented using large frame lengths can have a worse delay performance than the MW policy. This is expected because the analysis in Section II-D1 suggests that the average delay increases with the frame length.

For $N=3$ queues and $\epsilon=0.30$, Fig. 10 presents the average packet delay as a function of the sum throughput $\sum_{i} \lambda_{i}$ along the main diagonal line (i.e., $\lambda_{1}=\lambda_{2}=\lambda_{3}$ ). The maximum sum throughput is 0.65 as suggested by Lemma 2 . Similar to the previous case, Fig. 10 shows that the FBDC and the OLM policies stabilize the system for all $\sum_{i} \lambda_{i}<0.65$, and the system becomes unstable under the MW policy around $\sum_{i} \lambda_{i}=0.48$. This result also confirms that the OLM policy has a better delay performance than the other two policies.
The delay results in this section show that the OLM policy is not only simpler to implement as compared to the FBDC policy, but it can also be more delay efficient.

\section{CONCLUSION}

We investigated the dynamic server allocation problem with randomly varying connectivity and server switchover time. For the case of two queues, we analytically characterized the stability region of the system using state-action frequencies that are stationary solutions to an MDP formulation for the corresponding saturated system. We developed the throughput-optimal FBDC policy. We also developed simple myopic policies that achieve a large fraction of the stability region. We extended the stability region characterization in terms of state-action frequencies and the throughput optimality of the FBDC policy to the general system with arbitrary number of queues. We characterized tight analytical outer bounds on the stability region using an upper bound on the sum rate and showed that a simple GM policy achieves this sum-rate bound. The stability region characterization in terms of the state-action frequencies of the saturated system and the throughput optimality of the FBDC policy holds for systems with arbitrary switchover times and general Markovian channels. Furthermore, the FBDC policy provides a new framework for developing throughput-optimal policies for network control as this policy can be used to stabilize a large class of other network control problems.

In the future, we intend to explicitly characterize the stability region of systems with multiple-slot switchover times with general Markov modulated channels. We intend to develop throughput-optimal myopic policies for general system models. Finally, joint scheduling and routing in multihop networks with dynamically changing channels and server switchover times is a challenging future direction.

\section{APPENDIX A PROOF OF OBSERVATION 1}

In the following, we enumerate the states $s=\left(m, C_{1}, C_{2}\right)$ where $m$ denotes the queue the server is at, and $C_{1}$ and $C_{2}$ denote the states of the channel processes at each queue

$$
\begin{array}{llll}
(1,1,1) \equiv 1 & (1,1,0) \equiv 2 & (1,0,1) \equiv 3 & (1,0,0) \equiv 4 \\
(2,1,1) \equiv 5 & (2,1,0) \equiv 6 & (2,0,1) \equiv 7 & (2,0,0) \equiv 8
\end{array}
$$

Recall that $\mathbf{x}(s ; 1)$ and $\mathbf{x}(s ; 0)$ denote the state-action frequencies of being in state $s$ and choosing action stay and switch, respectively. We rewrite four of the eight balance equations of the state-action polytope $\mathbf{X}$ in open form in (21), shown at the bottom of this page. The time-average expected rates from the

$$
\begin{aligned}
& \mathbf{x}(1,1)+\mathbf{x}(1,0)=(1-\epsilon)^{2}(\mathbf{x}(1 ; 1)+\mathbf{x}(5 ; 0))+\epsilon(1-\epsilon)(\mathbf{x}(2 ; 1)+\mathbf{x}(6 ; 0))+\epsilon(1-\epsilon)(\mathbf{x}(3 ; 1)+\mathbf{x}(7 ; 0))+\epsilon^{2} \quad(\mathbf{x}(4 ; 1)+\mathbf{x}(8 ; 0)) \\
& \mathbf{x}(2,1)+\mathbf{x}(2,0)=\epsilon(1-\epsilon)(\mathbf{x}(1 ; 1)+x(5 ; 0))+(1-\epsilon)^{2}(\mathbf{x}(2 ; 1)+\mathbf{x}(6 ; 0))+\epsilon^{2} \quad(\mathbf{x}(3 ; 1)+\mathbf{x}(7 ; 0))+\epsilon(1-\epsilon)(\mathbf{x}(4 ; 1)+\mathbf{x}(8 ; 0)) \\
& \mathbf{x}(5,1)+\mathbf{x}(5,0)=(1-\epsilon)^{2}(\mathbf{x}(5 ; 1)+\mathbf{x}(1 ; 0))+\epsilon(1-\epsilon)(\mathbf{x}(6 ; 1)+\mathbf{x}(2 ; 0))+\epsilon(1-\epsilon)(\mathbf{x}(7 ; 1)+\mathbf{x}(3 ; 0))+\epsilon^{2} \quad(\mathbf{x}(8 ; 1)+\mathbf{x}(4 ; 0)) \\
& \mathbf{x}(7,1)+\mathbf{x}(7,0)=\epsilon(1-\epsilon)(\mathbf{x}(5 ; 1)+\mathbf{x}(1 ; 0))+\epsilon^{2} \quad(\mathbf{x}(6 ; 1)+\mathbf{x}(2 ; 0))+(1-\epsilon)^{2}(\mathbf{x}(7 ; 1)+\mathbf{x}(3 ; 0))+\epsilon(1-\epsilon)(\mathbf{x}(8 ; 1)+\mathbf{x}(4 ; 0))
\end{aligned}
$$


two queues $r_{1}(\mathbf{x})$ and $r_{2}(\mathbf{x})$ are given by $r_{1}(\mathbf{x})=\mathbf{x}(1 ; 1)+$ $\mathbf{x}(2 ; 1)$ and $r_{2}(\mathbf{x})=\mathbf{x}(5 ; 1)+\mathbf{x}(7 ; 1)$. Therefore, summing the expressions in (21), we obtain

$$
\begin{aligned}
\epsilon\left(r_{1}+r_{2}\right)= & (1-\epsilon)(\mathbf{x}(2 ; 0)+\mathbf{x}(7 ; 0))-\epsilon(\mathbf{x}(1 ; 0)+\mathbf{x}(5 ; 0)) \\
& +(1-\epsilon)(\mathbf{x}(3 ; 0)+\mathbf{x}(6 ; 0))+\epsilon(\mathbf{x}(7 ; 0)+\mathbf{x}(8 ; 0)) \\
& +\epsilon(\mathbf{x}(3 ; 1)+\mathbf{x}(4 ; 1)+\mathbf{x}(6 ; 1)+\mathbf{x}(8 ; 1)) .
\end{aligned}
$$

For the last term in this expression, we use the normalization condition in (6) to obtain

$$
\begin{aligned}
2 \epsilon\left(r_{1}+r_{2}\right)= & \epsilon-(\mathbf{x}(2 ; 0)+\mathbf{x}(7 ; 0))-2 \epsilon(\mathbf{x}(1 ; 0)+\mathbf{x}(5 ; 0)) \\
& +(1-2 \epsilon)(\mathbf{x}(3 ; 0)+\mathbf{x}(6 ; 0)) .
\end{aligned}
$$

From Corollary 1, there exists a stationary-deterministic policy $\pi$ that solves this LP of maximizing $r_{1}(\mathbf{x})+r_{2}(\mathbf{x})$ over the state-action polytope $\mathbf{X}$. Therefore, under this policy $\pi$, at each state, at least one of the actions must have 0 state-action frequency. From (22), we see that in order to maximize $r_{1}(\mathbf{x})+r_{2}(\mathbf{x}), \mathbf{x}(2 ; 0), \mathbf{x}(7 ; 0), \mathbf{x}(1,0), \mathbf{x}(5,0)$ need to be minimized $(\mathbf{x}(1,1), \mathbf{x}(5,1), \mathbf{x}(2 ; 1), \mathbf{x}(7 ; 1)$ need to be maximized $)$, and $\mathbf{x}(3,0), \mathbf{x}(6,0)$ need to be maximized $(\mathbf{x}(3,1), \mathbf{x}(6,1)$ need to be minimized). Therefore, we have $\mathbf{x}(1,0)=\mathbf{x}(2 ; 0)=$ $\mathbf{x}(5,0)=\mathbf{x}(7 ; 0)=\mathbf{x}(3,1)=\mathbf{x}(6 ; 1)=0$ in order to maximize $r_{1}(\mathbf{x})+r_{2}(\mathbf{x})$. The state-action frequencies corresponding to $\left(C_{1}, C_{2}\right)=(0,0)$, i.e., $\mathbf{x}(4 ; 1), \mathbf{x}(4 ; 0), \mathbf{x}(8 ; 1), \mathbf{x}(8 ; 0)$, do not affect the maximum achievable sum rate; however, different values of these frequencies correspond to moving on the maximum achievable sum-rate line (see Fig. 4). For instance, choosing queue 2 whenever the channel state is $\left(C_{1}, C_{2}\right)=(0,0)$, i.e., having $\mathbf{x}(4 ; 1)=\mathbf{x}(4 ; 0)=0$, gives the stationary-deterministic policy that corresponds to the upper corner of the sum-rate line in Fig. 4. Using these results in (22), we have

$$
2 \epsilon\left(r_{1}+r_{2}\right) \leq \epsilon+(1-2 \epsilon)(\mathbf{x}(3 ; 0)+\mathbf{x}(6 ; 0)) .
$$

Under the symmetric Gilbert-Elliot channel model, the steady-state probability of each channel state pair $\left(C_{1}, C_{2}\right)$ is $1 / 4$. Therefore, we have

$$
\begin{aligned}
& \mathbf{x}(1 ; 1)+\mathbf{x}(1 ; 0)+\mathbf{x}(5 ; 1)+\mathbf{x}(5 ; 0)=1 / 4 \\
& \mathbf{x}(2 ; 1)+\mathbf{x}(2 ; 0)+\mathbf{x}(6 ; 1)+\mathbf{x}(6 ; 0)=1 / 4 \\
& \mathbf{x}(3 ; 1)+\mathbf{x}(3 ; 0)+\mathbf{x}(7 ; 1)+\mathbf{x}(7 ; 0)=1 / 4 .
\end{aligned}
$$

Summing these expressions, we obtain

$$
r_{1}+r_{2} \leq 3 / 4-(\mathbf{x}(3 ; 0)+\mathbf{x}(6 ; 0)) .
$$

Combining this expression with (23) proves the result.

\section{APPENDIX B}

\section{GENERALIZATION TO NONSYMMETRIC GILBERT-ELLIOT CHANNELS}

In the following, we state results analogous to the results established in Section II for symmetric Gilbert-Elliot channels to the case of nonsymmetric Gilbert-Elliot channel model as given in Fig. 2.
Theorem 9: When the connectivity processes follow the nonsymmetric Gilbert-Elliot channel model, the rate region $\boldsymbol{\Lambda}_{s}$ is the set of all rates $r_{1} \geq 0, r_{2} \geq 0$ that for $p_{01}<\frac{\left(1-p_{10}\right)^{2}}{2-p_{10}}$ satisfy

$$
\begin{aligned}
p_{01} r_{1}+h_{1} r_{2} & \leq h_{1} \frac{p_{01}}{p_{01}+p_{10}} \\
\left(1-p_{10}\right) r_{1}+h_{2} r_{2} & \leq 1-\frac{p_{10}^{2}}{\left(p_{10}+p_{01}\right)^{2}}-\frac{p_{10} p_{01}}{p_{01}+p_{10}} \\
r_{1}+r_{2} & \leq 1-\frac{p_{10}^{2}}{\left(p_{10}+p_{01}\right)^{2}}-\frac{p_{10} p_{01}}{p_{01}+p_{10}} \\
h_{2} r_{1}+\left(1-p_{10}\right) r_{2} & \leq 1-\frac{p_{10}^{2}}{\left(p_{10}+p_{01}\right)^{2}}-\frac{p_{10} p_{01}}{p_{01}+p_{10}} \\
h_{1} r_{1}+p_{01} r_{2} & \leq h_{1} \frac{p_{01}}{p_{01}+p_{10}}
\end{aligned}
$$

where $h_{1}=\left(1-p_{01}\right)\left(1-p_{10}\right), h_{2}=1+p_{10}-p_{10}^{2}$, and for $p_{01} \geq \frac{\left(1-p_{10}\right)^{2}}{2-p_{10}}$ satisfy

$$
\begin{aligned}
p_{01} r_{1}+h_{3} r_{2} & \leq h_{3} \frac{p_{01}}{p_{10}+p_{01}} \\
r_{1}+r_{2} & \leq 1-\frac{p_{10}^{2}}{\left(p_{10}+p_{01}\right)^{2}}-\frac{p_{10} p_{01}}{p_{01}+p_{10}} \\
h_{3} r_{1}+p_{01} r_{2} & \leq h_{3} \frac{p_{01}}{p_{10}+p_{01}}
\end{aligned}
$$

where $h_{3}=\left(1-p_{10}\right)\left(p_{10}+\left(p_{10}+p_{01}\right)\left(1-p_{10}\right)\right)$.

The proof is lengthy and it is omitted here for brevity. It can be found in [14]. Closely examining the upper bound on sum rate $r_{1}+r_{2}$, the term $1-p_{10}^{2} /\left(p_{10}+p_{01}\right)^{2}$ is the steady-state probability that at least one channel is in ON state. This is the maximum achievable sum-rate value for the system with zero switchover delay studied in [39]. Therefore, the term $\frac{p_{10} p_{01}}{p_{01}+p_{10}}$ is exactly the loss due to switchover delay. It can be shown that, under a sum-rate-optimal policy, this term is equal to the steadystate probability that server is at a queue with an OFF channel state when the other queue is at an ON channel state.

The FBDC policy is asymptotically throughput optimal under the nonsymmetric Gilbert-Elliot channel model. This is straightforward as the FBDC policy only needs to solve the LP in Algorithm 2 for a given Markovian state transition structure, and the nonsymmetric Gilbert-Elliot channel model leads to a Markovian state transition structure. For the nonsymmetric Gilbert-Elliot channels case, the mappings from the queue sizes to the corner points of the rate region used by the FBDC policy, analogues to the mappings in Tables II and I, can be obtained from the slopes of the lines forming the boundary of the stability region. Furthermore, an analysis very similar to the one in Section II-E gives the corresponding mapping for the OLM policy. These mappings are shown in Table $\mathrm{V}$ for the case of $p_{01}<\frac{\left(1-p_{10}\right)^{2}}{2-p_{10}}$, and in Table VI for the case of $p_{01} \geq \frac{\left(1-p_{10}\right)^{2}}{2-p_{10}}$.

\section{APPENDIX C PROOF OF THEOREM 4}

Let $t_{k}$ be the first slot of the $k$ th frame where $t_{k+1}=t_{k}+T$. Let $D_{i}(t)$ be the service opportunity given to queue $i$ at time slot $t$, where $D_{i}(t)$ is equal to 1 if queue $i$ is scheduled at time 
TABLE V

Mapping From the Queue Sizes to the Corners of $\boldsymbol{\Lambda}_{\mathrm{s}}$, $b_{0}, b_{1}, b_{2}, b_{3}, b_{4}, b_{5}$, FOR $p_{01}<\frac{\left(1-p_{10}\right)^{2}}{2-p_{10}}$. FOR EACH STATE $\mathbf{s}=\left(m(t), C_{1}(t), C_{2}(t)\right)$, THE OPTIMAL ACTION IS SPECIFIED. THE THRESHOLDS ON $Q_{2} / Q_{1}$ FOR THE FBDC POLICY ARE

$0, T_{1}^{*}=p_{01} /\left(\left(1-p_{01}\right)\left(1-p_{10}\right)\right), T_{2}^{*}=\left(1-p_{10}\right) /\left(1+p_{10}-\right.$ $\left.p_{10}^{2}\right), 1, T_{3}^{*}=1 / T_{2}^{*}, T_{4}^{*}=1 / T_{1}^{*}$ AND FOR THE OLM POLICY ARE $0, T_{1}=p_{01} /\left(1-p_{10}\right), T_{2}=\left(1-p_{10}\right) /\left(2-p_{01}\right), 1, T_{3}=1 / T_{2}, T_{4}=$ $1 / T_{1}$. FOR EXAMPLE, CORNER $b_{1}$ IS CHOSEN IN THE FBDC POLICY IF $1 \leq Q_{2} / Q_{1}<T_{3}^{*}$, WHEREAS IN THE OLM POLICY IF $1 \leq Q_{2} / Q_{1}<T_{3}$

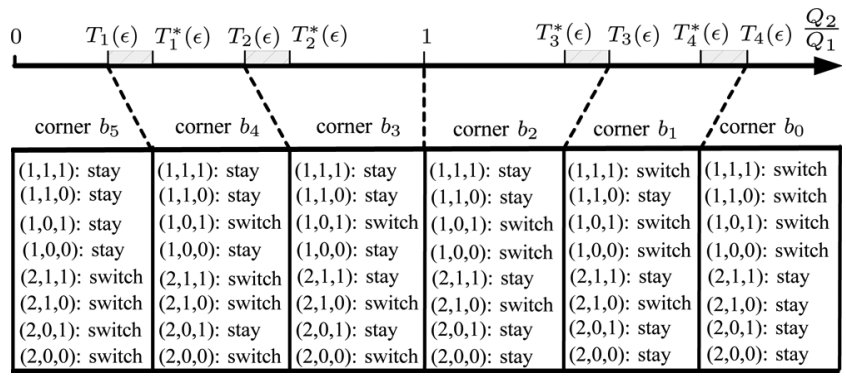

TABLE VI

MAPPING From THE QUEUE SizES TO THE CORNERS OF $\boldsymbol{\Lambda}_{\mathbf{s}}, b_{0}, b_{1}, b_{2}, b_{3}$, FOR $p_{01} \geq \frac{\left(1-p_{10}\right)^{2}}{2-p_{10}}$. FOR EACH STATE $\mathbf{s}=\left(m(t), C_{1}(t), C_{2}(t)\right)$, THE OPTIMAL ACTION IS SPECIFIED. THE THRESHOLDS ON $Q_{2} / Q_{1}$ FOR THE FBDC POLICY ARE $0, T_{1}^{*}=p_{01} /\left(\left(1-p_{10}\right)\left(p_{10}+\left(p_{10}+p_{01}\right)\left(1-p_{10}\right)\right)\right), 1, T_{2}^{*}$ $=\left(1-p_{10}\right)\left(p_{10}+\left(p_{10}+p_{01}\right)\left(1-p_{10}\right)\right) / p_{01}$ AND FOR THE OLM POLICY ARE $0, T_{1}=p_{01} /\left(1-p_{10}\right), 1, T_{2}=\left(1-p_{10}\right) / p_{01}$. FOR EXAMPLE, CORNER $b_{1}$ IS CHOSEN IN THE FBDC POLICY IF $1 \leq Q_{2} / Q_{1}<T_{2}^{*}$, WHEREAS IN THE OLM POLICY IF $1 \leq Q_{2} / Q_{1}<T_{2}$

\begin{tabular}{|c|c|c|c|}
\hline corner $b_{3}$ & corner $b_{2}$ & corner $b_{1}$ & corner $b_{0}$ \\
\hline $\begin{array}{l}(1,1,1): \text { stay } \\
(1,1,0): \text { stay } \\
(1,0,1): \text { stay } \\
(1,0,0): \text { stay } \\
(2,1,1): \text { switch } \\
(2,1,0): \text { switch } \\
(2,0,1): \text { switch } \\
(2,0,0): \text { switch }\end{array}$ & $\begin{array}{l}(1,1,1): \text { stay } \\
(1,1,0): \text { stay } \\
(1,0,1): \text { switch } \\
(1,0,0): \text { stay } \\
(2,1,1): \text { stay } \\
(2,1,0): \text { switch } \\
(2,0,1): \text { stay } \\
(2,0,0): \text { switch }\end{array}$ & $\begin{array}{l}(1,1,1): \text { stay } \\
(1,1,0): \text { stay } \\
(1,0,1): \text { switch } \\
(1,0,0): \text { switch } \\
(2,1,1): \text { stay } \\
(2,1,0): \text { switch } \\
(2,0,1): \text { stay } \\
(2,0,0): \text { stay }\end{array}$ & $\begin{array}{l}(1,1,1): \text { switch } \\
(1,1,0): \text { switch } \\
(1,0,1): \text { switch } \\
(1,0,0): \text { switch } \\
(2,1,1): \text { stay } \\
(2,1,0): \text { stay } \\
(2,0,1): \text { stay } \\
(2,0,0): \text { stay }\end{array}$ \\
\hline
\end{tabular}

slot $t$ (regardless of whether queue $i$ is empty or not) and zero otherwise. We have the following queue evolution relation:

$$
Q_{i}(t+1)=\max \left(Q_{i}(t)-D_{i}(t), 0\right)+A_{i}(t) .
$$

Similarly, the following $T$-step queue evolution relation holds:

$$
\begin{aligned}
Q_{i}\left(t_{k}+T\right) \leq \max & \left\{Q_{i}\left(t_{k}\right)-\sum_{\tau=0}^{T-1} D_{i}\left(t_{k}+\tau\right), 0\right\} \\
& +\sum_{\tau=0}^{T-1} A_{i}\left(t_{k}+\tau\right)
\end{aligned}
$$

where $\sum_{\tau=0}^{T-1} D_{i}\left(t_{k}+\tau\right)$ is the total service opportunity given to queue $i$ during the $k$ th frame. To see this, note that if $\sum_{\tau=0}^{T-1} D_{i}\left(t_{k}+\tau\right)$, the total service opportunity given to queue $i$ during the $k$ th frame, is smaller than $Q_{i}\left(t_{k}\right)$, then we have an equality. Otherwise, the first term is 0 and we have an inequality. This is because some of the arrivals during the frame might depart before the end of the frame. Note that $\sum_{\tau=0}^{T-1} D_{i}\left(t_{k}+\tau\right)$ denotes the link $i$ departures that would happen in the corresponding saturated system if we were to apply the same switching decisions over $T$ time slots in the corresponding saturated system. We first prove stability at the frame boundaries. Define the quadratic Lyapunov function

$$
L(\mathbf{Q}(t))=\sum_{i=1}^{2} Q_{i}^{2}(t)
$$

which represents a quadratic measure of the total load in the system at time slot $t$. Define the $T$-step conditional drift

$$
\Delta_{T}\left(t_{k}\right) \triangleq \mathbb{E}\left[L\left(\mathbf{Q}\left(t_{k}+T\right)\right)-L\left(\mathbf{Q}\left(t_{k}\right)\right) \mid \mathbf{Q}\left(t_{k}\right)\right]
$$

where the conditional expectation is over the randomness in arrivals and possibly the scheduling decisions. Squaring both sides of (25), using $\max (0, x)^{2} \leq x^{2}, \forall x \in \mathbb{N} \cup\{0\}$, and $D_{i}(t) \leq 1, \forall t$, we have

$$
\begin{aligned}
& Q_{i}\left(t_{k}+T\right)^{2}-Q_{i}\left(t_{k}\right)^{2} \leq T^{2}+\left(\sum_{\tau=0}^{T-1} A_{i}\left(t_{k}+\tau\right)\right)^{2} \\
& -2 Q_{i}\left(t_{k}\right)\left(\sum_{\tau=0}^{T-1} D_{i}\left(t_{k}+\tau\right)-\sum_{\tau=0}^{T-1} A_{i}\left(t_{k}+\tau\right)\right) .
\end{aligned}
$$

Summing (26) over the queues, using $\mathbb{E}\left[A_{i}(t)^{2}\right] \leq A_{\max }^{2}$ and $\mathbb{E}\left[A_{i}\left(t_{1}\right) A_{i}\left(t_{2}\right)\right] \leq \sqrt{\mathbb{E}\left[A_{i}\left(t_{1}\right)\right]^{2} \mathbb{E}\left[A_{i}\left(t_{2}\right)\right]^{2}} \leq \bar{A}_{\max }^{2}$ for all time slots $t_{1}$ and $t_{2}$, we can easily derive the following $T$-step conditional Lyapunov drift:

$$
\begin{aligned}
& \Delta_{T}\left(t_{k}\right) \leq 2 B T^{2}+2 T \sum_{i} Q_{i}\left(t_{k}\right) \lambda_{i} \\
& -2 \sum_{i} Q_{i}\left(t_{k}\right) \mathbb{E}\left[\sum_{\tau=0}^{T-1} D_{i}\left(t_{k}+\tau\right) \mid \mathbf{Q}\left(t_{k}\right)\right]
\end{aligned}
$$

where $B \doteq 1+A_{\max }^{2}$ and we used the fact that the arrival processes are i.i.d. over time, independent of the queue lengths. Recall the definition of the reward functions $\bar{r}_{i}\left(\mathbf{s}_{t}, \mathbf{a}_{t}\right)$ in (2) and (3) and let $\bar{r}_{i}\left(\mathbf{s}_{t}, \mathbf{a}_{t}\right)$ be the reward function associated with applying policy $\pi^{*}$ given in the definition of the FBDC policy in Algorithm 2 to the saturated system. Let $\bar{r}_{i}(t)$ denote $\bar{r}_{i}\left(\mathbf{s}_{t}, \mathbf{a}_{t}\right)$ for notational simplicity, $i \in\{1,2\}$. Note that $r_{i}(t)$ is equal to $D_{i}(t)$, since $D_{i}(t)$ is the service opportunity given to link $i$ at time slot $t$. Now, let $\mathbf{r}^{*}=\left(r_{i}^{*}\right)_{i}$ be the infinite horizon average rate associated with policy $\pi^{*}$. Let $\mathbf{x}^{*}$ be the optimal vector of state-action frequencies corresponding to $\pi^{*}$. Define the time-average empirical reward from queue $i$ in the saturated system, $\hat{r}_{T, i}\left(t_{k}\right), i \in\{1,2\}$ by

$$
\hat{r}_{T, i}\left(t_{k}\right) \doteq \frac{1}{T} \sum_{\tau=0}^{T-1} \bar{r}_{i}\left(t_{k}+\tau\right) .
$$

Similarly, define the time-average empirical state-action frequency vector $\hat{\mathbf{x}}_{T}\left(t_{k} ; \mathbf{s}, \mathbf{a}\right)$

$$
\hat{\mathbf{x}}_{T}\left(t_{k} ; \mathbf{s}, \mathbf{a}\right) \doteq \frac{1}{T} \sum_{\tau=t_{k}}^{t_{k}+T-1} I_{\left\{\mathbf{s}_{\tau}=\mathbf{s}, \mathbf{a}_{\tau}=\mathbf{a}\right\}}
$$


where $I_{E}$ is the indicator function of an event $E$, i.e., $I_{E}=1$ if $E$ occurs and $I_{E}=0$ otherwise. Using the definition of the reward functions in (2) and (3), we have that

$$
\hat{r}_{T, i}\left(t_{k}\right)=\sum_{\mathbf{s} \in \mathcal{S}} \sum_{\mathbf{a}} \bar{r}_{i}(\mathbf{s}, \mathbf{a}) \hat{\mathbf{x}}_{T}\left(t_{k} ; \mathbf{s}, \mathbf{a}\right), i \in\{1,2\}
$$

and $\hat{\mathbf{r}}_{T}\left(t_{k}\right)=\left(\hat{r}_{T, 1}\left(t_{k}\right)\right)_{i}$. Similarly, we have

$$
r_{i}^{*}=\sum_{\mathbf{s} \in \mathcal{S}} \sum_{\mathbf{a}} \bar{r}_{i}(\mathbf{s}, \mathbf{a}) \mathbf{x}^{*}(\mathbf{s}, \mathbf{a}), i \in\{1,2\}
$$

Now, we utilize the following key MDP theory result in Lemma 4.1 [26], which states that as $T$ increases, $\hat{\mathbf{x}}_{T}\left(t_{k}\right)=\left(\hat{\mathbf{x}}_{T}\left(t_{k} ; \mathbf{s}, \mathbf{a}\right)\right)_{\mathbf{s}, \mathbf{a}}$ converges to $\mathbf{x}^{*}$.

Lemma 3: For every choice of initial state distribution, there exists constants $c_{1}$ and $c_{2}$ such that

$$
\mathbb{P}\left(\left\|\hat{\mathbf{x}}_{T}\left(t_{k}\right)-\mathbf{x}^{*}\right\| \geq \delta_{0}\right) \leq c_{1} e^{-c_{2} \delta_{0}^{2} T}, \quad \forall T \geq 1, \forall \delta_{0}>0 .
$$

Furthermore, convergence of $\hat{\mathbf{x}}_{T}\left(t_{k}\right)$ to $\mathbf{x}^{*}$ is w.p. 1 .

This result applies in our system because every extreme point $\mathbf{x}^{*}$ of $\mathbf{X}$ can be attained by a stationary and deterministic policy that has a single irreducible recurrent class in its underlying Markov chain [26], [34]. ${ }^{4}$ Due to the linear mapping from the state-action frequencies to the rewards, by Schwartz inequality, each component of $\hat{\mathbf{r}}_{T}\left(t_{k}\right)$ also converges to the corresponding component of $\mathbf{r}^{*}$. Therefore, we have that for every choice of initial state distribution, there exists constants $c_{1}$ and $c_{2}$ such that

$$
\mathbb{P}\left(\left\|\hat{\mathbf{r}}_{T}\left(t_{k}\right)-\mathbf{r}^{*}\right\| \geq \delta_{1}\right) \leq c_{1} e^{-c_{2} \delta_{1}^{2} T}, \forall T \geq 1, \forall \delta_{1}>0 .
$$

Furthermore, convergence of $\hat{\mathbf{r}}_{T}\left(t_{k}\right)$ to $\mathbf{r}^{*}$ is with probability 1 . Now, let $R_{T}\left(t_{k}\right) \doteq \sum_{i} Q_{i}\left(t_{k}\right) \hat{r}_{T, i}\left(t_{k}\right)$ and $R^{*}\left(t_{k}\right) \doteq \sum_{i} Q_{i}\left(t_{k}\right) r_{i}^{*}$. We rewrite the drift expression

$$
\begin{aligned}
\frac{\Delta_{T}\left(t_{k}\right)}{2 T} \leq B T & +\sum_{i} Q_{i}\left(t_{k}\right) \lambda_{i}-\mathbb{E}\left[R_{T}\left(t_{k}\right) \mid \mathbf{Q}\left(t_{k}\right)\right] \\
=B T & +\sum_{i} Q_{i}\left(t_{k}\right) \lambda_{i}-\sum_{i} Q_{i}\left(t_{k}\right) r_{i}^{*} \\
& +\mathbb{E}\left[R^{*}\left(t_{k}\right)-R_{T}\left(t_{k}\right) \mid \mathbf{Q}\left(t_{k}\right)\right] .
\end{aligned}
$$

Now, we bound the last term. For all $\delta_{2}>0$, we have

$$
\begin{aligned}
& \mathbb{E}\left[R^{*}\left(t_{k}\right)-R_{T}\left(t_{k}\right) \mid \mathbf{Q}\left(t_{k}\right)\right]= \\
& =\mathbb{E}\left[R^{*}\left(t_{k}\right)-R_{T}\left(t_{k}\right) \mid \mathbf{Q}\left(t_{k}\right), R^{*}\left(t_{k}\right)-R_{T}\left(t_{k}\right) \geq \delta_{2}\left\|\mathbf{Q}\left(t_{k}\right)\right\|\right] \\
& \quad . \mathbb{P}\left(R^{*}\left(t_{k}\right)-R_{T}\left(t_{k}\right) \geq \delta_{2}\left\|\mathbf{Q}\left(\mathbf{t}_{\mathbf{k}}\right)\right\| \mid \mathbf{Q}\left(t_{k}\right)\right) \\
& +\mathbb{E}\left[R^{*}\left(t_{k}\right)-R_{T}\left(t_{k}\right) \mid \mathbf{Q}\left(t_{k}\right), R^{*}\left(t_{k}\right)-R_{T}\left(t_{k}\right)<\delta_{2}\left\|\mathbf{Q}\left(t_{k}\right)\right\|\right] \\
& \quad . \mathbb{P}\left(R^{*}\left(t_{k}\right)-R_{T}\left(t_{k}\right)<\delta_{2}\left\|\mathbf{Q}\left(\mathbf{t}_{\mathbf{k}}\right)\right\| \mid \mathbf{Q}\left(t_{k}\right)\right) \\
& \leq\left(\sum_{i} Q_{i}\left(t_{k}\right)\right) \mathbb{P}\left(\left|R^{*}\left(t_{k}\right)-R_{T}\left(t_{k}\right)\right| \geq \delta_{2}\left\|\mathbf{Q}\left(t_{k}\right)\right\| \mathbf{Q}\left(t_{k}\right)\right) \\
& \quad+\delta_{2}\left\|\mathbf{Q}\left(t_{k}\right)\right\|
\end{aligned}
$$

${ }^{4}$ Note that, in general, multiple stationary-deterministic policies can yield the same optimal reward vector $\mathbf{r}^{*}$. Among these, we choose the one that forms a Markov chain with a single recurrent class. where we bound the first expectation by $\sum_{i} Q_{i}\left(t_{k}\right)$ by using $\left\|\mathbf{r}^{*}\right\|<1$, the second expectation by $\delta_{2}\left\|\mathbf{Q}\left(t_{k}\right)\right\|$, and the second probability by 1 . By Schwartz inequality, we have

$$
\begin{gathered}
\mathbb{P}\left(\left|R^{*}\left(t_{k}\right)-R_{T}\left(t_{k}\right)\right| \geq \delta_{2}|| \mathbf{Q}\left(\mathbf{t}_{\mathbf{k}}\right) \| \mid \mathbf{Q}\left(t_{k}\right)\right) \\
\leq \mathbb{P}\left(\left\|\mathbf{r}^{*}-\hat{\mathbf{r}}_{T}\left(t_{k}\right)\right\| \geq \delta_{2} \mid \mathbf{Q}\left(t_{k}\right)\right) .
\end{gathered}
$$

Using (29) and (32) in (31), we have

$$
\mathbb{E}\left[R^{*}\left(t_{k}\right)-R_{T}\left(t_{k}\right) \mid \mathbf{Q}\left(t_{k}\right)\right] \leq\left(\sum_{i} Q_{i}\left(t_{k}\right)\right) c_{1} e^{-c_{2} \delta_{2}^{2} T}+\delta_{2}\left\|\mathbf{Q}\left(t_{k}\right)\right\| .
$$

Hence, using $\left\|\mathbf{Q}\left(t_{k}\right)\right\| \leq \sum_{i} Q_{i}\left(t_{k}\right)$, we bound (30) as

$$
\begin{aligned}
\frac{\Delta_{T}\left(t_{k}\right)}{2 T} \leq B T & +\sum_{i} Q_{i}\left(t_{k}\right) \lambda_{i}-\sum_{i} Q_{i}\left(t_{k}\right) r_{i}^{*} \\
& +\left(\sum_{i} Q_{i}\left(t_{k}\right)\right)\left(c_{1} e^{-c_{3} \delta_{2}^{2} T}+\delta_{2}\right) .
\end{aligned}
$$

Therefore, calling $\delta \doteq c_{1} e^{-c_{3} \delta_{2}^{2} T}+\delta_{2}$, we have

$$
\frac{\Delta_{T}\left(t_{k}\right)}{2 T} \leq B T+\sum_{i} Q_{i}\left(t_{k}\right) \lambda_{i}-\sum_{i} Q_{i}\left(t_{k}\right) r_{i}^{*}+\delta \sum_{i} Q_{i}\left(t_{k}\right) \text {. }
$$

Now for $\lambda$ strictly inside the $\delta$-stripped stability region $\Lambda_{s}^{\delta}$, there exist a small $\xi>0$ such that $\lambda+\xi .1=\mathbf{r}-\delta \mathbf{1}$, for some $\mathbf{r} \in \boldsymbol{\Lambda}_{s}$. Utilizing this and the fact that $\sum_{i} Q_{i}(t)\left(r_{i}-r_{i}^{*}\right) \leq 0$ by definition of the FBDC policy in Algorithm 2, we have

$$
\frac{\Delta_{T}\left(t_{k}\right)}{2 T} \leq B T-\left(\sum_{i} Q_{i}\left(t_{k}\right)\right) \xi
$$

Therefore, the queue sizes have negative drift when $\sum_{i} Q_{i}\left(t_{k}\right)$ is larger than $B T / \xi$. This establishes stability of the queue sizes at the frame boundaries $t=k T, k=\{0,1,2, \ldots\}$ for $\lambda$ within the $\delta$-stripped stability region $\Lambda_{s}^{\delta}[14]$.

For any given time $t \in\left(t_{k}, t_{k+1}\right)$, we have $Q_{i}(t) \leq$ $Q_{i}\left(t_{k}\right)+\sum_{\tau=0}^{T-1} A_{i}\left(t_{k}+\tau\right)$. Therefore, $\mathbb{E}\left[Q_{i}(t)\right] \leq$ $\mathbb{E}\left[Q_{i}\left(t_{k}\right)\right]+T \lambda_{i} \leq \mathbb{E}\left[Q_{i}\left(t_{k}\right)\right]+T A_{\max }$. Hence, stability at the frame boundaries implies the overall stability of the system. Finally, $\delta=c_{1} e^{-c_{3} \delta_{2}^{2} T}+\delta_{2}$ for any $\delta_{2}>0$. Therefore, choosing $\delta_{2}$ appropriately (for example, $\delta_{2}=T^{-0.5+\delta_{3}}$ for some small $\delta_{3}>0$ ), we have that $\delta(T)$ is a decreasing function of $T$. Therefore, for any $\delta>0$, one can find $T$ such that the hypothesis of the theorem holds.

\section{APPENDIX D \\ PROOF OF THEOREM 5}

We first show that the OLM policy produces a mapping from the set of queue sizes to the stationary-deterministic policies corresponding to the corners of the stability region. This mapping is similar to that of the FBDC policy; however, the thresholds on the queue size ratios $Q_{2} / Q_{1}$ are determined according to (13).

Mapping from queue sizes to actions. Case-1: $\epsilon<\epsilon_{c}$

For $\epsilon<\epsilon_{c}$, there are six corners in the stability region denoted by $b_{0}, b_{1}, \ldots, b_{5}$ where $b_{0}$ is $(0,0.5)$ and $b_{5}$ is $(0.5,0)$ as shown in Fig. 4(a). We derive conditions on $Q_{2} / Q_{1}$ such that the OLM policy chooses the stationary-deterministic decisions that correspond to a given corner point.

Corner $b_{0}$ :

As can be seen from Table I, the actions corresponding to corner $b_{0}$ are to stay at queue 2 for every channel condition, i.e., the server chooses queue 2 even when the channel states are 
$\left(C_{1}(t), C_{2}(t)\right)=(1,0)$. Therefore, using (13), for the myopic policy to take the deterministic actions corresponding to $b_{0}$, we must have

$$
Q_{1}(1-\epsilon) \leq Q_{2}(\epsilon) \Rightarrow \frac{Q_{2}}{Q_{1}} \geq \frac{1-\epsilon}{\epsilon} .
$$

This means that if we apply the myopic policy with coefficients $Q_{1}, Q_{2}$ such that $Q_{2} / Q_{1} \geq(1-\epsilon) / \epsilon$, then the system output rate will be driven toward the corner point $b_{0}$.

Corner $b_{1}$ :

As shown in Table I, the deterministic actions corresponding to corner $b_{1}$ are as follows: At queue 1, stay only if the channel states $\left(C_{1}, C_{2}\right)$ are $(1,0)$, and at queue 2 , switch only if the channel states are $(1,0)$. For the OLM policy to take these eight deterministic actions, $Q_{2} / Q_{1}$ needs to satisfy the conditions for all the eight actions under switching decisions according to (13). The most limiting conditions are switching at $\left(C_{1}, C_{2}\right)=(1,1)$ at queue 1 and switching at $\left(C_{1}, C_{2}\right)=(1,0)$ at queue 2 . The conditions on $Q_{2} / Q_{1}$ for the OLM policy to take these actions are $Q_{1}(2-\epsilon)<Q_{2}(1-\epsilon)$ and $Q_{1}(1-\epsilon)>Q_{2} \epsilon$. Combining these, we have

$$
\frac{2-\epsilon}{1-\epsilon} \leq \frac{Q_{2}}{Q_{1}}<\frac{1-\epsilon}{\epsilon} .
$$

Note that the condition $\epsilon<\epsilon_{c}=1-\sqrt{2} / 2$ implies that $\left.\frac{1-\epsilon}{\epsilon}\right\rangle$ $\frac{2-\epsilon}{1-\epsilon}$.

\section{Corner $b_{2}$ :}

As shown in Table I, the deterministic actions corresponding to corner $b_{2}$ are as follows: At queue 1, stay only if the channel states $\left(C_{1}, C_{2}\right)$ are $(1,0)$ or $(1,1)$, and at queue 2 , switch only if the channel states are $(1,0)$. The most limiting actions are switching at $\left(C_{1}, C_{2}\right)=(1,0)$ at queue 2 , staying at $\left(C_{1}, C_{2}\right)=$ $(1,1)$ at queue 1 , and switching at $\left(C_{1}, C_{2}\right)=(0,0)$ at queue 1 . The conditions on $Q_{2} / Q_{1}$ for the OLM policy to take these actions are $Q_{1}(1-\epsilon)>Q_{2} \epsilon, Q_{1}(2-\epsilon)>Q_{2}(1-\epsilon)$, and $Q_{1} \leq Q_{2}$, respectively. Combining these and noting that since $\epsilon \geq \epsilon_{c}$ we have $\frac{1-\epsilon}{\epsilon} \geq \frac{2-\epsilon}{1-\epsilon}$, we obtain the intersection of all the conditions given by

$$
1 \leq \frac{Q_{2}}{Q_{1}}<\frac{2-\epsilon}{1-\epsilon} .
$$

The conditions for the corners $b_{3}, b_{4}$, and $b_{3}$ are symmetric, completing the mapping from the queue sizes to the corners of $\boldsymbol{\Lambda}_{\mathbf{S}}$ for $\epsilon \geq \epsilon_{c}$ which is shown in Table III. This mapping is in general different from the corresponding mapping of the FBDC policy in Table I.

Mapping from queue sizes to actions. Case-2: $\epsilon \geq \epsilon_{c}$

In this case, there are four corner points in the throughput region, where the corners $b_{0}, b_{2}, b_{3}, b_{5}$ are the same as the case $\epsilon<\epsilon_{c}$, and the corners $b_{1}$ and $b_{4}$ for the case $\epsilon<\epsilon_{c}$ are dominated when we have $\epsilon \geq \epsilon_{c}$.

Corner $b_{0}$ :

The analysis for the corner $b_{0}$ is the same as that for the corner $b_{0}$ in the previous case. Therefore, for the myopic policy to take the deterministic actions corresponding to the corner $b_{0}$, we need

$$
\frac{Q_{2}}{Q_{1}}>\frac{1-\epsilon}{\epsilon} .
$$

Corner $b_{2}$ :

The analysis is very similar to the analysis for the corner point $b_{0}$ for the case $\epsilon<\epsilon_{c}$. As shown in Table II, the deterministic actions corresponding to corner $b_{2}$ are as follows: At queue 1 , stay only if the channel states $\left(C_{1}, C_{2}\right)$ are $(1,0)$ or $(1,1)$, and at queue 2 , switch only if the channel states are $(1,0)$. The most limiting actions are switching at $\left(C_{1}, C_{2}\right)=(1,0)$ at queue 2 , staying at $\left(C_{1}, C_{2}\right)=(1,1)$ at queue 1 , and switching at $\left(C_{1}, C_{2}\right)=(0,0)$ at queue 1 . The conditions on $Q_{2} / Q_{1}$ for the OLM policy to take these actions are $Q_{1}(1-\epsilon)>Q_{2} \epsilon$, $Q_{1}(2-\epsilon)>Q_{2}(1-\epsilon)$, and $Q_{1} \leq Q_{2}$, respectively. Because we have $\epsilon \geq \epsilon_{c}$ in this case, we have $\frac{1-\epsilon}{\epsilon}<\frac{2-\epsilon}{1-\epsilon}$. Therefore, combining the conditions, we have

$$
1 \leq \frac{Q_{2}}{Q_{1}}<\frac{1-\epsilon}{\epsilon} .
$$

The conditions for the corners $b_{3}$ and $b_{5}$ are symmetric, completing the mapping from the queue sizes to the corners of $\boldsymbol{\Lambda}_{\mathbf{S}}$ for $\epsilon \geq \epsilon_{c}$ which is shown in Table IV. Again, this mapping is in general different from the corresponding mapping of the FBDC policy in Table II. Therefore, for a given ratio of the queue sizes $Q_{2} / Q_{1}$, the FBDC and the OLM policies may apply different stationary-deterministic policies corresponding to different corner points of $\boldsymbol{\Lambda}_{\mathbf{s}}$, denoted by $\mathbf{r}^{*}$ and $\hat{\mathbf{r}}$, respectively. The shaded intervals of $Q_{2} / Q_{1}$ in Tables III and IV are the intervals in which the OLM and the FBDC policies apply different policies.

Next, using these mappings, Lemma 4 given at the end of this section shows that the weighted average departure rate of the OLM policy is at least $90 \%$ of that of the FBDC policy

$$
\Psi \doteq \frac{\sum_{i} Q_{i}(t) \hat{r}_{i}}{\sum_{i} Q_{i}(t) r_{i}^{*}} \geq 90 \%
$$

We finalize the proof of Theorem 5 by showing that the bound on $\Psi$ in (35) is a sufficient condition for the OLM policy to achieve at least $90 \%$ of $\boldsymbol{\Lambda}_{\mathrm{s}}$ asymptotically in $T$. The following drift expression for the OLM policy can be derived similarly to the derivation of (33) used in the proof of Theorem 4

$$
\frac{\Delta_{T}\left(t_{k}\right)}{2 T} \leq B T+\sum_{i} Q_{i}\left(t_{k}\right) \lambda_{i}-\sum_{i} Q_{i}\left(t_{k}\right) \hat{r}_{i}+\delta_{4} \sum_{i} Q_{i}\left(t_{k}\right)
$$

where $\delta_{4}(T)$ is a decreasing function of $T$. Using (34)

$$
\frac{\Delta_{T}\left(t_{k}\right)}{2 T} \leq B T+\sum_{i} Q_{i}\left(t_{k}\right) \lambda_{i}-0.9 \sum_{i} Q_{i}\left(t_{k}\right) r_{i}^{*}+\delta_{4} \sum_{i} 90 Q_{i}\left(t_{k}\right) .
$$

Using an argument similar to that for (34), we have that for $\left(\lambda_{1}, \lambda_{2}\right)$ strictly inside the 0.9 fraction of the $\delta_{4}$-stripped stability region, there exist a small $\xi>0$ such that $\left(\lambda_{1}, \lambda_{2}\right)+(\xi, \xi)=0.9\left(r_{1}, r_{2}\right)-\left(\delta_{4}, \delta_{4}\right)$, for some $\mathbf{r}=\left(r_{1}, r_{2}\right) \in \boldsymbol{\Lambda}_{\mathbf{s}}$. Substituting this expression for $\left(\lambda_{1}, \lambda_{2}\right)$ and using $\sum_{i} Q_{i}(t)\left(r-r_{i}^{*}\right) \leq 0$, we have

$$
\begin{aligned}
& \frac{\Delta_{T}\left(t_{k}\right)}{T} \leq B T+0.9 \sum_{i} Q_{i}\left(t_{k}\right)\left(r-r_{i}^{*}\right) \\
& \quad-\left(\sum_{i} Q_{i}\left(t_{k}\right)\right) \delta_{4}-\left(\sum_{i} Q_{i}\left(t_{k}\right)\right) \xi+\left(\sum_{i} Q_{i}\left(t_{k}\right)\right) \delta_{4} .
\end{aligned}
$$


After cancellations, we have

$$
\frac{\Delta_{T}\left(t_{k}\right)}{2 T} \leq B T-\left(\sum_{i} Q_{i}\left(t_{k}\right)\right) \xi
$$

Therefore, using an argument similar to the proof of Theorem 4 in Appendix $\mathrm{C}$, the system is stable for arrival rates within at least the 0.9 fraction of $\delta_{4}$-stripped stability region, where $\delta_{4}(T)$ is a decreasing function of $T$. This establishes the proof of Theorem 5 .

Lemma 4: We have that

$$
\Psi \doteq \frac{\sum_{i} Q_{i}(t) \hat{r}_{i}}{\sum_{i} Q_{i}(t) r_{i}^{*}} \geq 90 \% \text {. }
$$

Proof: Considering the mappings in Tables III and IV, for the regions of $\epsilon$ where the OLM policy and the optimal policy "choose" the same corner point, we have $\Psi=1$. In the following, we analyze the ratio $\Psi$ in the regions where the two policies choose different corner points, which we call "discrepant" regions. We will use $Q_{1}$ and $Q_{2}$ instead of $Q_{1}(t)$ and $Q_{2}(t)$ for notational simplicity. We first consider the case $Q_{2}>Q_{1}$, and divide the proof into separate cases for different regions of $\epsilon$ values.

Weighted Departure-Rate Ratio Analysis, Case 1: $\epsilon<\epsilon_{c}$

Note that the following inequality always holds: $\frac{2-\epsilon}{1-\epsilon}>$ $\frac{\left(1+\epsilon-\epsilon^{2}\right)}{(1-\epsilon)}$. However, we have $\frac{2-\epsilon}{1-\epsilon}=\frac{(1-\epsilon)^{2}}{\epsilon}$ for $\epsilon=\epsilon_{t} \doteq 0.245$ for the case of $\epsilon<\epsilon_{c}=0.293$.

Case 1.1: $\epsilon<\epsilon_{t}$

For this case, we have $\frac{2-\epsilon}{1-\epsilon}<\frac{(1-\epsilon)^{2}}{\epsilon}$.

Discrepant Region 1: $\frac{(1-\epsilon)^{2}}{\epsilon}<\frac{Q_{2}}{Q_{1}}<\frac{1-\epsilon}{\epsilon}$

In this case, the OLM policy chooses the corner point $b_{1}$, whereas the optimal policy chooses the corner point $b_{0}$. Therefore

$$
\begin{aligned}
\Psi & =\frac{Q_{1}\left(\frac{(1-\epsilon)^{2}}{4}\right)+Q_{2}\left(\frac{1}{2}-\frac{\epsilon}{4}\right)}{Q_{2} \frac{1}{2}} \geq 1-\frac{\epsilon}{2}+\frac{(1-\epsilon)^{2}}{2} \frac{\epsilon}{1-\epsilon} \\
& =1-\frac{\epsilon^{2}}{2} \geq 0.9700 .
\end{aligned}
$$

Discrepant Region 2: $\frac{\left(1+\epsilon-\epsilon^{2}\right)}{1-\epsilon}<\frac{Q_{2}}{Q_{1}}<\frac{2-\epsilon}{1-\epsilon}$

In this case, the OLM policy chooses the corner point $b_{2}$, whereas the optimal policy chooses the corner point $b_{1}$. Therefore

$$
\begin{aligned}
\Psi & =\frac{Q_{1}\left(\frac{3}{8}-\frac{\epsilon}{2}+\frac{\epsilon}{8(2-\epsilon)}\right)+Q_{2}\left(\frac{3}{8}-\frac{\epsilon}{8(2-\epsilon)}\right)}{Q_{1}\left(\frac{(1-\epsilon)^{2}}{4}\right)+Q_{2}\left(\frac{1}{2}-\frac{\epsilon}{4}\right)} \\
& =\frac{\frac{3}{8}-\frac{\epsilon}{2}+\frac{\epsilon}{8(2-\epsilon)}+\frac{Q_{2}}{Q_{1}}\left(\frac{3}{8}-\frac{\epsilon}{8(2-\epsilon)}\right)}{\frac{(1-\epsilon)^{2}}{4}+\frac{Q_{2}}{Q_{1}}\left(\frac{1}{2}-\frac{\epsilon}{4}\right)} \geq 0.9002 .
\end{aligned}
$$

This is a minimization of a function of two variables for all possible $\epsilon$ values in the interval $0 \leq \epsilon \leq \epsilon_{t}$, and the ratio $\frac{Q_{2}}{Q_{1}}$ in the interval $\frac{\left(1+\epsilon-\epsilon^{2}\right)}{1-\epsilon}<\frac{Q_{2}}{Q_{1}}<\frac{2-\epsilon}{1-\epsilon}$.

Case 1.2: $\epsilon_{t}<\epsilon<\epsilon_{c}$

For this case, we have $\frac{2-\epsilon}{1-\epsilon} \geq \frac{(1-\epsilon)^{2}}{\epsilon}$

Discrepant Region 1: $\frac{(2-\epsilon)}{(1-\epsilon)}<\frac{Q_{2}}{Q_{1}}<\frac{1-\epsilon}{\epsilon}$
In this case, the OLM policy chooses the corner point $b_{1}$, whereas the optimal policy chooses the corner point $b_{0}$. Therefore

$$
\begin{aligned}
\Psi & =\frac{Q_{1}\left(\frac{(1-\epsilon)^{2}}{4}\right)+Q_{2}\left(\frac{1}{2}-\frac{\epsilon}{4}\right)}{Q_{2} \frac{1}{2}} \geq 1-\frac{\epsilon}{2}+\frac{(1-\epsilon)^{2}}{2} \frac{\epsilon}{1-\epsilon} \\
& =1-\frac{\epsilon^{2}}{2} \geq 0.9500 .
\end{aligned}
$$

Discrepant Region 2: $\frac{(1-\epsilon)^{2}}{\epsilon}<\frac{Q_{2}}{Q_{1}}<\frac{2-\epsilon}{1-\epsilon}$

In this case, the OLM policy chooses the corner point $b_{2}$, whereas the optimal policy chooses the corner point $b_{0}$. Therefore

$$
\begin{aligned}
\Psi & =\frac{Q_{1}\left(\frac{3}{8}-\frac{\epsilon}{2}+\frac{\epsilon}{8(2-\epsilon)}\right)+Q_{2}\left(\frac{3}{8}-\frac{\epsilon}{8(2-\epsilon)}\right)}{Q_{2} \frac{1}{2}} \\
& \geq\left(\frac{1-\epsilon}{2-\epsilon}\right)\left(\frac{3}{4}-\epsilon+\frac{\epsilon}{4(2-\epsilon)}\right)+\frac{3}{4}-\frac{\epsilon}{4(2-\epsilon)} \geq 0.9150 .
\end{aligned}
$$

Discrepant Region 3: $\frac{\left(1+\epsilon-\epsilon^{2}\right)}{1-\epsilon}<\frac{Q_{2}}{Q_{1}}<\frac{(1-\epsilon)^{2}}{\epsilon}$

In this case, the OLM policy chooses the corner point $b_{2}$, whereas the optimal policy chooses the corner point $b_{1}$. Therefore

$$
\begin{aligned}
\Psi & =\frac{Q_{1}\left(\frac{3}{8}-\frac{\epsilon}{2}+\frac{\epsilon}{8(2-\epsilon)}\right)+Q_{2}\left(\frac{3}{8}-\frac{\epsilon}{8(2-\epsilon)}\right)}{Q_{1}\left(\frac{(1-\epsilon)^{2}}{4}\right)+Q_{2}\left(\frac{1}{2}-\frac{\epsilon}{4}\right)} \\
& \geq \frac{\frac{3}{8}-\frac{\epsilon}{2}+\frac{\epsilon}{8(2-\epsilon)}+\frac{Q_{2}}{Q_{1}}\left(\frac{3}{8}-\frac{\epsilon}{8(2-\epsilon)}\right)}{\frac{(1-\epsilon)^{2}}{4}+\frac{Q_{2}}{Q_{1}}\left(\frac{1}{2}-\frac{\epsilon}{4}\right)} \geq 0.9474 .
\end{aligned}
$$

Due to symmetry, the same bounds on $\Psi$ applies for $Q_{2}<Q_{1}$. Weighted Departure-Rate Ratio Analysis, Case 2: $\epsilon \geq \epsilon_{c}$

For the case where $\epsilon \geq \epsilon_{c}$, we have $(1-\epsilon)(3-2 \epsilon) \leq(1-\epsilon) / \epsilon$ and $\frac{1-\epsilon}{\epsilon}<\frac{2-\epsilon}{1-\epsilon}$. Therefore, the only discrepant region between the FBDC and the OLM policies for $Q_{2}>Q_{1}$ is given by $(1-\epsilon)(3-2 \epsilon) \leq \frac{Q_{2}}{Q_{1}}<\frac{1-\epsilon}{\epsilon}$, where for this interval, the OLM policy chooses the corner point $b_{1}$, whereas the FBDC policy chooses the corner point $b_{0}$.

Discrepant Region 1: $(1-\epsilon)(3-2 \epsilon)<\frac{Q_{2}}{Q_{1}}<\frac{1-\epsilon}{\epsilon}$

In this case, the OLM policy chooses the corner point $b_{1}$, whereas the optimal policy chooses the corner point $b_{0}$. Therefore

$$
\begin{aligned}
\Psi & =\frac{Q_{1}\left(\frac{3}{8}-\frac{\epsilon}{2}+\frac{\epsilon}{8(2-\epsilon)}\right)+Q_{2}\left(\frac{3}{8}-\frac{\epsilon}{8(2-\epsilon)}\right)}{Q_{2} \frac{1}{2}} \\
& \geq\left(\frac{\epsilon}{1-\epsilon}\right)\left(\frac{3}{4}-\epsilon+\frac{\epsilon}{4(2-\epsilon)}\right)+\frac{3}{4}-\frac{\epsilon}{4(2-\epsilon)} \geq 0.914 .
\end{aligned}
$$

Due to symmetry, the same bound on $\Psi$ applies for $Q_{2}<Q_{1}$. Combining all the cases, for all $\epsilon \in[0,0.5]$, we have that $\Psi \geq$ 0.90 for all possible $Q_{1}$ and $Q_{2}$.

\section{APPENDIX E}

PROOF OF LEMMA 2

We follow similar steps to the proof of the sum-throughput upper bound for the case of two queues in Appendix A. In order to obtain an expression for $r_{i}, i \in\{1, \ldots, N\}$, we sum the $2^{N-1}$ equations in (16) for which the server location $m$ is $i$ 
and the channel process of queue $i, C_{i}$, is 1 . This gives for all $i \in\{1, \ldots, N\}$

$$
\begin{aligned}
p_{10} r_{i}= & -\sum_{\substack{s: m=i \\
C_{i}=1}} \sum_{a \neq i} \mathbf{x}(s, a)+p_{01} \sum_{\substack{s: m=i \\
\dot{C}_{i}=0}} \mathbf{x}(s ; i) \\
& +\left(1-p_{10}\right) \sum_{\substack{s: m \neq i \\
C_{i}=1}} \mathbf{x}(s ; i)+p_{01} \sum_{\substack{s: m \neq i \\
C_{i}=0}} \mathbf{x}(s ; i) .
\end{aligned}
$$

Summing $r_{i}$ over all queues and using the normalization condition $\sum_{s} \sum_{a} \mathbf{x}(s, a)=1$, we have

$$
\begin{aligned}
\left(p_{10}+p_{01}\right) \sum_{i=1}^{N} r_{i} & =p_{01}-\sum_{i=1}^{N} \sum_{j \neq i} \sum_{\substack{s: m=i \\
C_{i}=1, C_{j}=0}} \mathbf{x}(s ; j) \\
& -\left(p_{01}+p_{10}\right) \sum_{i=1}^{N} \sum_{j \neq i} \sum_{\substack{j: m=i \\
C_{i}=1, \bar{C}_{j}=1}} \mathbf{x}(s ; j) \\
& +\left(1-p_{01}-p_{10}\right) \sum_{i=1}^{N} \sum_{j \neq i} \sum_{\substack{s: m=i \\
C_{i}=0, \bar{C}_{j}=1}} \mathbf{x}(s ; j) .
\end{aligned}
$$

From Corollary 1, there exists a stationary-deterministic policy $\pi$ that solves this LP of maximizing $\sum_{i} r_{i}(\mathbf{x})$ over the stateaction polytope $\mathbf{X}$. Therefore, under this policy $\pi$, at each state, at least one of the actions must have 0 state-action frequency. Therefore, in order to maximize the sum rate, the terms that have negative contribution to the sum rate must be zero

$\left(p_{10}+p_{01}\right) \sum_{i=1}^{N} r_{i}=p_{01}+\left(1-p_{01}-p_{10}\right) \sum_{i=1}^{N} \sum_{j \neq i} \sum_{\substack{s: m=i \\ C_{i}=0, \bar{C}_{j}=1}} \mathbf{x}(s ; j)$.

Similar to the two-queue case in Appendix A, we utilize the expressions resulting from the fact that the steady-state probability of each channel state vector is known. For instance, for $C_{0}^{(N)} \doteq \mathbb{P}\left(\left(C_{1}, \ldots, C_{N}\right)=(0, \ldots, 0)\right)=\frac{p_{10}^{N}}{\left(p_{10}+p_{01}\right)^{N}}$, we have

$$
\sum_{i=1}^{N} \sum_{\substack{s: m=i \\ C_{j}=0, \forall j}} \sum_{a \in A} \mathbf{x}(i, a)=C_{0}^{(N)}
$$

Summing these expressions, we obtain

$$
\sum_{i=1}^{N} \sum_{j \neq i} \sum_{\substack{s: m=i \\ C_{i}=0, \bar{C}_{j}=1}} \mathbf{x}(s ; j)=1-C_{0}^{(N)}-\sum_{i=1}^{N} r_{i} .
$$

Combining this expression with (36), we obtain

$$
\sum_{i=1}^{N} r_{i}=1-C_{0}^{(N)}-\left(p_{10}\left(1-C_{0}^{(N)}\right)-p_{01} C_{0}^{(N)}\right) .
$$

\section{REFERENCES}

[1] S. Ahmad, L. Mingyan, T. Javidi, Q. Zhao, and B. Krishnamachari, "Optimality of myopic sensing in multichannel opportunistic access," IEEE Trans. Inf. Theory, vol. 55, no. 9, pp. 4040-4050, Sep. 2009.

[2] S. Ahmad and M. Liu, "Multi-channel opportunistic access: A case of restless bandits with multiple plays," in Proc. 47th Annu. Allerton Conf. Commun., Control, Comput., Oct. 2009, pp. 1361-1368.
[3] I. F. Akyildiz and X. Wang, Wireless Mesh Networks. New York: Wiley, 2009.

[4] E. Altman, Constrained Markov Decision Processes. London, U.K.: Chapman \& Hall, 1999.

[5] E. Altman and A. Shwartz, "Markov decision problems and state-action frequencies," SIAM J. Control Optim., vol. 29, no. 4, pp. 786-809, Jul. 1991.

[6] E. Altman, P. Konstantopoulos, and Z. Liu, "Stability, monotonicity and invariant quantities in general polling systems," Queuing Syst., vol. 11, pp. 35-57, Mar. 1992.

[7] E. Altman and H. J. Kushner, "Control of polling in presence of vacations in heavy traffic with applications to satellite and mobile radio systems," SIAM J. Control Optim., vol. 41, pp. 217-252, 2002.

[8] D. Bertsimas and J. Tsitsiklis, "Introduction to Linear Optimization," Athena Scientific. Belmont, MA, 1997.

[9] L. Blake and M. Long, "Antennas: Fundamentals, Design, Measurement," SciTech. Raleigh, NC, 2009.

[10] O. J. Boxma, W. P. Groenendijk, and J. A. Weststrate, "A pseudoconservation law for service systems with a polling table," IEEE Trans. Commun., vol. 38, no. 10, pp. 1865-1870, Oct. 1990.

[11] A. Brzezinski and E. Modiano, "Dynamic Reconfiguration and routing algorithms for IP-over-WDM networks with stochastic traffic," $J$. Lightw. Technol., vol. 23, no. 10, pp. 3188-3205, Oct. 2005.

[12] P. Chaporkar, K. Kar, and S. Sarkar, "Throughput guarantees through maximal scheduling in wireless networks," in Proc. 43rd Annu. Allerton Conf. Commun., Control Comput., Sep. 2005, pp. 28-30.

[13] G. D. Çelik, L. B. Le, and E. Modiano, "Scheduling in parallel queues with randomly varying connectivity and switchover delay," in Proc. IEEE Int. Conf. Comput. Commun., Apr. 2011, pp. 316-320.

[14] G. D. Celik, L. B. Le, and E. Modiano, Dynamic server allocation over time-varying channels with switchover delay 2012, arXiv Tech. Rep. 1203.0696

[15] G. C , S. Borst, P. Whiting, and E. Modiano, "Variable frame based max-weight algorithms for networks with switchover delay," in Proc. IEEE Int. Symp. Inf. Theory, Aug. 2011, pp. 2537-2541.

[16] Y. Chen, Q. Zhao, and A. Swami, "Joint design and separation principle for opportunistic spectrum access in the presence of sensing errors," IEEE Trans. Inf. Theory, vol. 54, no. 5, pp. 2053-2071, May 2008.

[17] A. Eryilmaz, A. Ozdaglar, and E. Modiano, "Polynomial complexity algorithms for full utilization of multi-hop wireless networks," in Proc. 26th IEEE Int. Conf. Comput. Commun., May 2007, pp. 499-507.

[18] L. Georgiadis, M. Neely, and L. Tassiulas, Resource Allocation and Cross-Layer Control in Wireless Networks. Hanover, MA: Now Publishers, 2006

[19] E. N. Gilbert, "Capacity of burst-noise channels," Bell Syst. Tech. J., vol. 39, pp. 1253-1265, Sep. 1960.

[20] K. Kar, X. Luo, and S. Sarkar, "Throughput-optimal scheduling in multichannel access point networks under infrequent channel measurements," in Proc. IEEE Int. Conf. Comput. Commun., May 2007, pp. $1640-1648$.

[21] L. B. Le, E. Modiano, C. Joo, and N. B. Shroff, "Longest-queue-first scheduling under SINR interference model," in Proc. 11 th ACM Symp. Mobile Ad hoc Netw. Comput., Sep. 2010, pp. 41-50.

[22] H. Levy, M. Sidi, and O. L. Boxma, "Dominance relations in polling systems," Queueing Syst., vol. 6, pp. 155-172, Apr. 1990.

[23] C. Li and M. Neely, "On achievable network capacity and throughputachieving policies over Markov ON/OFF channels," presented at the WiOpt, Jun. 2010.

[24] X. Lin and N. B. Shroff, "The impact of imperfect scheduling on cross-layer rate control in wireless networks," in Proc. IEEE Int. Conf. Comput. Commun., Mar. 2005, pp. 1804-1814.

[25] Z. Lui, P. Nain, and D. Towsley, "On optimal polling policies," Queuing Syst., vol. 11, pp. 59-83, Jul. 1992.

[26] S. Mannor and J. N. Tsitsiklis, "On the empirical state-action frequencies in Markov decision processes under general policies," Math. Oper. Res., vol. 30, no. 3, pp. 545-561, Aug. 2005.

[27] E. Modiano and R. Barry, "A novel medium access control protocol for WDM-based LAN's and access networks using a master/slave scheduler," J. Lightw. Technol., vol. 18, no. 4, pp. 461-468, Apr. 2000.

[28] E. Modiano, D. Shah, and G. Zussman, "Maximizing throughput in wireless networks via gossip," in Proc. ACM SIGMETRICS/Performance, Jun. 2006, pp. 27-38.

[29] M. J. Neely, "Stochastic network optimization with application to communication and queueing systems," Morgan \& Claypool. San Rafael, CA, 2010. 
[30] M. J. Neely, "Stochastic optimization for Markov modulated networks with application to delay constrained wireless scheduling," in Proc. 48th IEEE Conf. Decis. Control, Dec. 2009, pp. 4826-4833.

[31] M. J. Neely, E. Modiano, and C. E. Rohrs, "Dynamic power allocation and routing for time varying wireless networks," IEEE J. Sel. Areas Commun., vol. 23, no. 1, pp. 89-103, Jan. 2005.

[32] M. J. Neely, E. Modiano, and C. E. Rohrs, "Power allocation and routing in multi-beam satellites with time varying channels," IEEE Trans. Netw., vol. 11, no. 1, pp. 138-152, Feb. 2003.

[33] M. J. Neely, E. Modiano, and C. E. Rohrs, "Tradeoffs in delay guarantees and computation complexity in $\mathrm{n} \times \mathrm{n}$ packet switches," presented at the presented at the Conf. Inf. Sci. Syst., Princeton, NJ, Mar. 2002.

[34] M. Puterman, Markov Decision Processes: Discrete Stochastic Dynamic Programming. New York: Wiley, 2005.

[35] D. Shah and D. J. Wischik, "Optimal scheduling algorithms for inputqueued switches," in Proc. IEEE Int. Conf. Comput. Commun., Mar. 2006, pp. 1-11.

[36] A. Pantelidou, A. Ephremides, and A. Tits, "A cross-layer approach for stable throughput maximization under channel state uncertainty," Wireless Netw., vol. 15, no. 5, pp. 555-569, Jul. 2009.

[37] A. L. Stolyar, "Maxweight scheduling in a generalized switch: State space collapse and workload minimization in heavy traffic," Ann. Appl. Prob., vol. 14, no. 1, pp. 1-53, 2004.

[38] L. Tassiulas and A. Ephremides, "Stability properties of constrained queueing systems and scheduling policies for maximum throughput in multihop radio networks," IEEE Trans. Autom. Control, vol. 37, no. 12, pp. 1936-1948, Dec. 1992.

[39] L. Tassiulas and A. Ephremides, "Dynamic server allocation to parallel queues with randomly varying connectivity," IEEE Trans. Inf. Theory, vol. 39, no. 2, pp. 466-478, Mar. 1993.

[40] A. Tolkachev, V. Denisenko, A. Shishlov, and A. Shubov, "High gain antenna systems for millimeter wave radars with combined electronical and mechanical beam steering," in Proc. IEEE Int. Symp. Phased Array Syst. Technol., Oct. 2006, pp. 266-271.

[41] V. M. Vishnevskii and O. V. Semenova, "Mathematical methods to study the polling systems," Autom. Remote Control., vol. 67, no. 2, pp. 173-220, Feb. 2006.

[42] H. Wang and P. Chang, "On verifying the first-order Markovian assumption for a Rayleigh fading channel model," IEEE Trans. Veh. Technol., vol. 45, no. 2, pp. 353-357, May 1996.

[43] X. Wu and R. Srikant, "Bounds on the capacity region of multi-hop wireless networks under distributed greedy scheduling," presented at the presented at the IEEE Int. Conf. Comput. Commun., Barcelona, Spain, Mar. 2006.

[44] L. Ying and S. Shakkottai, "On throughput-optimality with delayed network-state information," in Proc. Inf. Theory Appl. Workshop, Jan. 2008, pp. 339-344.

[45] Q. Zhao, B. Krishnamachari, and K. Liu, "On myopic sensing for multichannel opportunistic access: Structure, optimality, and performance," IEEE Trans. Wireless Commun., vol. 7, no. 12, pp. 5431-5440, Dec. 2008.

[46] M. Zorzi, R. Rao, and L. Milstein, "On the accuracy of a first-order Markov model for data transmission on fading channels," in Proc. 4th IEEE Int. Conf. Universal Pers. Commun., 1995, pp. 211-215.

Güner D.Celik (S'06) received his B.S. degree in Electrical and Electronics Engineering from the Middle East Technical University (METU), Ankara, Turkey with second highest ranking in 2005, and his S.M. degree in Electrical Engineering and Computer Science Department (EECS) from the Massachusetts Institute of Technology (MIT) in 2007. In August 2010, he was a visiting researcher at Bell Labs in Mathematics of Networks and Complex Systems Research Group in NJ and in summer 2007, he joined Microsoft Research (MSR) Systems and Networking Group in Cambridge, UK as a research intern. He is currently pursuing a Ph.D. degree in Laboratory For Information and Decision Systems (LIDS) in the EECS department at MIT under the supervision of Prof. Eytan Modiano. His research interests are in wireless network control, queuing theory and communication theory. In particular, he is currently working on scheduling, routing and resource allocation for network control and optimization, dynamic server scheduling in queuing systems and trajectory control for data gathering in mobile wireless networks.

Long B. Le (S'04-M'07) received the B.Eng. (with Highest Distinction) degree from Ho Chi Minh City University of Technology, Vietnam, in 1999, the M.Eng. degree from Asian Institute of Technology, Pathumthani, Thailand, in 2002, and the Ph.D. degree from the University of Manitoba, Winnipeg, MB, Canada, in 2007.

From 2008 to 2010, he was a postdoctoral research associate with Massachusetts Institute of Technology, Cambridge, MA. Since 2010, he has been an assistant professor with the Institut National de la Recherche Scientifique (INRS), Universite du Quebec, Montreal, QC, Canada, where he leads a research group working on cognitive radio and dynamic spectrum sharing, radio resource management, network control and optimization.

Dr. Le is a member of the editorial board of IEEE Wireless Communications Letters and IEEE Communications Surveys and Tutorials. He has served as technical program committee co-chairs of the New Results track at IEEE VTC2012-Fall, Wireless Networks track at IEEE VTC2011-Fall, and the Cognitive Radio and Spectrum Management track at IEEE PIMRC2011.

Eytan Modiano (S'90-M'93-SM'00-F'12) received his B.S. degree in Electrical Engineering and Computer Science from the University of Connecticut at Storrs in 1986 and his M.S. and Ph.D. degrees, both in Electrical Engineering, from the University of Maryland, College Park, MD, in 1989 and 1992 respectively. He was a Naval Research Laboratory Fellow between 1987 and 1992 and a National Research Council Post Doctoral Fellow during 1992-1993. Between 1993 and 1999 he was with MIT Lincoln Laboratory where he was a project leader for MIT Lincoln Laboratory's Next Generation Internet (NGI) project. Since 1999, he has been on the faculty at MIT; where he is a Professor in the Department of Aeronautics and Astronautics and the Laboratory for Information and Decision Systems (LIDS). His research is on communication networks and protocols with emphasis on satellite, wireless, and optical networks. He is the co-recipient of the Sigmetrics 2006 Best paper award for the paper "Maximizing Throughput in Wireless Networks via Gossiping," and the Wiopt 2005 best student paper award for the paper "Minimum Energy Transmission Scheduling Subject to Deadline Constraints."

$\mathrm{He}$ is currently an Associate Editor for IEEE/ACM TRANSACTIONS ON NETWORKING. He had served as Associate Editor for IEEE TRANSACTIONS ON INFORMATION THEORY, and as guest editor for IEEE JSAC special issue on WDM network architectures; the Computer Networks Journal special issue on Broadband Internet Access; the Journal of Communications and Networks special issue on Wireless Ad-Hoc Networks; and for IEEE Journal of Lightwave Technology special issue on Optical Networks. He was the Technical Program co-chair for IEEE Wiopt 2006, IEEE Infocom 2007, and ACM MobiHoc 2007. 